\title{
Research Article \\ On Chebyshev's Systems and Non-Uniform Sampling Related to Caputo Fractional Dynamic Time-Invariant Systems
}

\author{
M. De la Sen \\ Institute for Research and Development of Processes, Faculty of Science and Technology, \\ University of Basque Country, Campus of Leioa, Aptdo. 544, 48080 Bilbao, Spain \\ Correspondence should be addressed to M. De la Sen, manuel.delasen@ehu.es
}

Received 4 October 2010; Accepted 18 December 2010

Academic Editor: Jinde Cao

Copyright (C) 2010 M. De la Sen. This is an open access article distributed under the Creative Commons Attribution License, which permits unrestricted use, distribution, and reproduction in any medium, provided the original work is properly cited.

\begin{abstract}
This paper is concerned with the investigation of the controllability and observability of Caputo fractional differential linear systems of any real order $\alpha$. Expressions for the expansions of the evolution operators in powers of the matrix of dynamics are first obtained. Sets of linearly independent continuous functions or matrix functions, which are also Chebyshev's systems, appear in such expansions in a natural way. Based on the properties of such functions, the controllability and observability of the Caputo fractional differential system of real order $\alpha$ are discussed as related to their counterpart properties in the corresponding standard system defined for $\alpha=1$. Extensions are given to the fulfilment of those properties under non-uniform sampling. It is proved that the choice of the appropriate sampling instants is not restrictive as a result of the properties of the associate Chebyshev's systems.
\end{abstract}

\section{Introduction}

Caputo fractional calculus is a very useful tool to calculate alternative solutions to the classical ones in many applications as, for instance, in dynamic systems (see, e.g., [1-3]). Since the fractional order $\alpha$ can be a nonpositive integer, even real or complex, the technique can be used to better fix the trajectory solution of mathematical models to obtained experimental data due to modelling or measuring errors. On the other hand, the so-called Chebyshev system of $n$ linearly independent functions in the Banach space of continuous functions $C([a, b])$, endowed with the supremum norm, has the important property, due to Haar, that each nontrivial polynomial of this system has at most $(n-1)$ distinct zeros in $[a, b],[4]$. 
There are many sets of linearly independent functions which are Chebyshev's systems as, for instance, (a) the sets $\left\{t^{k}: k=0, \ldots, n-1\right\}$, respectively, $\{\cos k t: k=0, \ldots, n-1\}$ on any real interval $[a, b]$ of nonzero finite measure, respectively, on $[0,2 \pi]$, and $(b)$ the set $\left\{f(t) t^{k}: k=0, \ldots, n-1\right\}$ with $f(t)$ being continuous and with no zeros on $[a, b]$ is a Chebyshev system on $[a, b]$. The linearly independent functions defining the expansions of the $C_{0}$-semigroup $\left\{e^{A t}: t \in \mathbf{R}_{0+}\right\}$, of differential generator $A$, in powers of $A$ are a Chebyshev system on any interval $[\gamma, \gamma+\pi / \omega)$ where $\omega$ is the maximum eigenfrequency; that is, the maximum absolute imaginary part of any complex eigenvalues, if any, and otherwise $\omega=+\infty$ (see [5-7], firstly discussed in [6]). In [5], it is proven that the property holds even if $A$ is a complex matrix which is not related by a similarity transformation to some real one, that is, some potential complex eigenvalues may not have their complex conjugate counterparts as eigenvalues. In $[6,7]$, the Haar property of Chebyshev systems is used to formulate the following properties:

(a) controllability to the origin of standard linear time-invariant dynamic systems (roughly speaking, the ability to steer any initial condition to the equilibrium in any finite time by injecting some admissible control) under, in general, non-uniform (also referred to as aperiodic or nonperiodic) sampling,

(b) observability of standard linear time-invariant dynamic (i.e., the ability to calculate initial conditions from past values of a measured output trajectory) under, in general, non-uniform sampling,

(c) identifiability of standard linear dynamic time-invariant systems (i.e., the ability to compute the values of the parameters from output measurements and eventually time-derivatives up till some order of the output trajectory) and model matching of linear and time-invariant systems also under, in general, non-uniform sampling.

It has been proven in the above papers that the Haar property ensures that the corresponding properties are transferred from the continuous-time dynamic system to its discrete-time counterpart for infinitely many choices of sets of sufficient large cardinal (exceeding a lowerbound being dependent on the degree of the minimal polynomial of $A$ and the dimensions of the output and input spaces) of distinct sampling instants. The particular choices of the sampling instants may be done by some practical considerations as, for instance, to achieve a well-conditioned coefficient matrix of the resulting algebraic problem related to controllability, observability, local identifiability and so forth. The study of the properties of controllability, reachability (related to controllability to any final state), observability and constructability (closely related to observability) under non-uniform sampling has been extended more recently to nonfractional positive linear continuous time systems, namely, those having nonnegative state and output trajectory for any given non-negative initial conditions and controls [8]. Many other theoretical studies and applications have been performed. In [9], the reconstruction problem from non-uniform data is focused on. In [10], the general non-uniform sampling in a stochastic framework is investigated. The problem of sequential estimation under non-uniform sampling is studied in [11]. In [12-14], some estimation properties and application under non-uniform sampling are described. The sampling efficiency in event-based sampling laws of signals is discussed in [15]. Finally, some filtering properties under non-uniformly sampled signals are investigated in [16]. All those commented studies were referred to the standard (i.e., nonfractional) case. Therefore, the 
extension given in this manuscript which includes the fractional case of positive real order is novel in the context of non-uniform sampling. The paper is organized as follows. Section 2 is devoted to some preliminary results concerning the expansions of the evolution operators of the solution in powers of the matrix of dynamics of a linear time-invariant dynamic system. Sets of Chebyshev's systems of functions appear in a natural way in such expansions. Such sets have the property of keeping nonsingular, under very generic sampling, the coefficient matrices of the algebraic systems of equations associate to controllability, respectively, observability problems in the case that the continuous-time counterpart is controllable or, respectively, observable. The results are extended in Section 3 for Caputo fractional dynamic systems on any real order $\alpha$. Those results are used in Sections 4-5 to discuss the properties of observability and controllability and their counterparts under non-uniform sampling. Finally, an example is given in Section 6.

Some notations used are $\mathbf{R}_{0+}:=\mathbf{R}_{+} \cup\{0\}=\left\{z \in \mathbf{R}_{+}: z \geq 0\right\} ; \mathbf{R}_{+}:=\left\{z \in \mathbf{R}_{+}: z>0\right\}$. A corresponding notation $\mathbf{Z}_{0+}, \mathbf{Z}_{+}$are used for the corresponding subsets of the integer set $\mathbf{Z}$. $\bar{n}:=\{1,2, \ldots, n\}$, for all $n \in \mathbf{Z}_{+}$, and $A>0$ denotes that the matrix $A$ is positive definite.

\section{Preliminaries on the Expansions of $\exp (\mathbf{A t})$ and Associate Chebyshev's Systems}

This section is devoted to preliminary basic results about useful sets of linearly independent real functions and real matrix functions of time, which are also Chebyshev systems [4-7], and used for the expansions of the evolution operators defining the solution of the differential system.

Lemma 2.1. Assume that $\mu$ is the degree of the minimal polynomial of $A \in \mathbf{C}^{n \times n}$. Then $\left\{A^{i} \in \mathbf{C}^{n \times n}\right.$ : $i \in \overline{\mu-1} \cup\{0\}\}$ is a linearly independent set of matrices.

Proof. Proceed by contradiction by assuming that the set $\left\{A^{i} \in \mathrm{C}^{n \times n}: i \in \overline{\mu-1} \cup\{0\}\right\}$ is linearly dependent so that there is a nonidentically zero set $\left\{\lambda_{i} \in \mathrm{C}: i \in \overline{\mu-1} \cup\{0\}\right\}$ such that $\sum_{i=1}^{\mu-1} \lambda_{i} A^{i}=0$. Assume that $\lambda_{j} \neq 0$ for $j \in \overline{\mu-1} \cup\{0\}$ so that

$$
A^{j}=-\lambda_{j}^{-1}\left(\sum_{i(<j)=1}^{\mu-1} \lambda_{i} A^{i}+\sum_{i(>j)}^{\mu-1} \lambda_{i} A^{i}\right) .
$$

Choose $j=\max \left(i \in \overline{\mu-1} \cup\{0\}: \lambda_{k}=0, \forall k(>i) \in \overline{\mu-1} \cup\{0\}\right)$ which always exists since $\left\{\lambda_{i} \in\right.$ $\mathrm{C}: i \in \overline{\mu-1} \cup\{0\}\}$ is a nonidentically zero set and $0 \leq j \leq \mu-1$. Thus, $A^{j}=-\sum_{i(<j)=1}^{\mu-1} \lambda_{j}^{-1} \lambda_{i} A^{i}$ and some $0 \leq j \leq \mu-1$ is then the degree of the minimal polynomial of $A$ which contradicts that such a degree is $\mu$.

Lemma 2.2. The following formula $A^{p}=\sum_{k=0}^{\mu-1} a_{k}(p) A^{k}$ holds with unique complex coefficients $a_{k}(p)$, for all $k \in \overline{\mu-1} \cup\{0\}$, for all $p\left(\in \mathbf{Z}_{+}\right) \geq \mu$. 
Proof. Note from Cayley-Hamilton theorem that $A^{\mu}=\sum_{k=0}^{\mu-1} a_{k}(\mu) A^{k}$, thus,

$$
\begin{aligned}
A^{\mu+1} & =\sum_{k=0}^{\mu-1} a_{k}(\mu) A^{k+1}=\sum_{k=0}^{\mu-2} a_{k}(\mu) A^{k+1}+a_{\mu-1} A^{\mu} \\
& =\sum_{k=1}^{\mu-1} a_{k-1}(\mu) A^{k}+a_{\mu-1}(\mu)\left(\sum_{k=0}^{\mu-1} a_{k}(\mu) A^{k}\right) \\
& =\sum_{k=0}^{\mu-1}\left(a_{k-1}(\mu)+a_{k}(\mu) a_{\mu-1}(\mu)\right) A^{k}=\sum_{k=0}^{\mu-1} a_{k}(\mu+1) A^{k},
\end{aligned}
$$

so that the above identity holds irrespective of $A$ if and only if

$a_{k}(\mu+1)=a_{\mu-1}(\mu) a_{k}(\mu)+a_{k-1}(\mu)=\frac{a_{0}(\mu+1)}{a_{0}(\mu)} a_{k}(\mu)+a_{k-1}(\mu) ; \quad \forall k \in \overline{\mu-1} \cup\{0\}, a_{-1}(\mu)=0$.

Proceeding recursively with the above formulas, one gets for all $\mathbf{Z}_{+} \ni p \geq 1$

$$
\begin{aligned}
A^{\mu+p} & =\sum_{k=0}^{\mu-1} a_{k}(\mu+p-1) A^{k+1}=\sum_{k=0}^{\mu-1}\left(a_{k-1}(\mu+p-1)+a_{k}(\mu+p-1) a_{\mu-1}(\mu+p-i-1)\right) A^{k} \\
& =\sum_{k=0}^{\mu-1} a_{k}(\mu+p) A^{k}
\end{aligned}
$$

with

$$
a_{k}(\mu+p)=a_{\mu-1}^{p}(\mu) a_{k}(\mu)+\sum_{i=0}^{p-1} a_{\mu-1}^{i}(\mu) a_{k-1}(\mu+p-i-1) .
$$

The uniqueness of the coefficients follows from Lemma 2.1 as follows. Assume that there are two sets of coefficients such that $A^{p}=\sum_{k=0}^{\mu-1} a_{k}(p) A^{k}=\sum_{k=0}^{\mu-1} a_{k}^{\prime}(p) A^{k}$. Then, $\sum_{k=0}^{\mu-1}\left(a_{k}(p)-\right.$ $\left.a_{k}^{\prime}(p)\right) A^{k}=0$ which implies $a_{k}(p)=a_{k}^{\prime}(p)$ for all $k \in \overline{p-1} \cup\{0\}$ for all $p\left(\in \mathbf{Z}_{+}\right) \geq \mu$ from Lemma 2.1 since $\left\{A^{i} \in \mathrm{C}^{n \times n}: i \in \overline{\mu-1} \cup\{0\}\right\}$ is a linearly independent set.

\section{Alternative Proof}

Equivalently, proceed by complete induction by assuming that

$$
A^{\mu+j}=\sum_{k=0}^{\mu-1} a_{k}(\mu) A^{k+j}=\sum_{k=0}^{\mu-1} a_{k}(\mu+j-1) A^{k+1} ; \quad \forall j \in \overline{p-1}
$$


with

$$
a_{k}(\mu+j)=a_{\mu-1}^{j}(\mu) a_{k}(\mu)+\sum_{i=0}^{j-1} a_{\mu-1}^{i}(\mu) a_{k-1}(\mu+j-i-1) ; \quad \forall j \in \overline{p-1}
$$

Then, it follows that (2.4) holds with

$$
\begin{aligned}
& a_{k}(\mu+j) \\
& =a_{\mu-1}(\mu+j-1) a_{k}(\mu+j-1)+a_{k-1}(\mu+j-1) \\
& =a_{\mu-1}^{j}(\mu) a_{k}(\mu)+\sum_{i=0}^{j-1} a_{\mu-1}^{i}(\mu) a_{k-1}(\mu+j-i-1) ; \quad \forall k \in \overline{\mu-1} \cup\{0\}, a_{-1}(j)=0, \forall j \in \overline{p-1} .
\end{aligned}
$$

A particular case of interest of $(2.8)$ is

$$
\begin{aligned}
a_{k}(n) & =a_{\mu-1}(n-1) a_{k}(n-1)+a_{k-1}(n-1) \\
& =a_{\mu-1}^{n-\mu}(\mu) a_{k}(\mu)+\sum_{i=0}^{n-\mu-1} a_{\mu-1}^{i}(\mu) a_{k-1}(n-i-1) ; \quad \forall k \in \overline{\mu-1} \cup\{0\}, a_{-1}(n-1)=0
\end{aligned}
$$

that allows to interpret the two following common formulas for the Cayley-Hamilton theorem:

$$
A^{\mu}=\sum_{k=0}^{\mu-1} a_{k}(\mu) A^{k} ; \quad A^{n}=\sum_{k=0}^{\mu-1} a_{k}(n) A^{k}
$$

where $\mu \leq n$ and $n$ are, respectively, the degrees of the minimal and characteristic polynomials of $A$. On the other hand, it is possible to extend the expansion of powers of $A$ up till the degree of the characteristic polynomial of $A$ through the use of modified coefficients $\bar{a}_{k}(\cdot)$ as follows:

$$
\begin{aligned}
A^{n} & =\sum_{k=0}^{n-1} \bar{a}_{k}(n) A^{k}=\sum_{k=0}^{\mu-1} a_{k}(\mu) A^{k} \\
& =\sum_{k=0}^{\mu-1} \bar{a}_{k}(n) A^{k}+\sum_{k=\mu}^{n-1} \bar{a}_{k}(n) A^{k}=\sum_{k=0}^{\mu-1} \bar{a}_{k}(n) A^{k}+\sum_{k=0}^{n-1-\mu} \bar{a}_{k+\mu}(n) A^{k+\mu} \\
& =\sum_{k=0}^{\mu-1} \bar{a}_{k}(n) A^{k}+\sum_{k=0}^{n-1-\mu} \bar{a}_{k+\mu}(n)\left(\sum_{j=0}^{\mu-1} a_{j}(\mu+k) A^{j}\right) \\
& =\sum_{j=0}^{\mu-1}\left(\bar{a}_{j}(n)+\sum_{k=0}^{n-1-\mu} \bar{a}_{k+\mu}(n) a_{j}(\mu+k)\right) A^{j}
\end{aligned}
$$


so that $\bar{a}_{j}(n)=a_{j}(\mu)-\sum_{k=\mu}^{n-1} \bar{a}_{k}(n) a_{j}(k)$, for all $k \in \overline{n-1} \cup\{0\}$ and it turns out that the extension also works for $A^{p}$, for all $p\left(\in \mathbf{Z}_{+}\right) \geq \mu$ with $\bar{a}_{k}(\mu)=a_{k}(\mu)$, for all $k \in \overline{p-1} \cup\{0\}$, and

$$
\bar{a}_{k}(p)=a_{k}(\mu)-\sum_{j=\mu}^{p-1} \bar{a}_{j}(p) a_{k}(k) ; \quad \forall k \in \overline{p-1} \cup\{0\}
$$

for $p \geq \mu+1$. There has been proven the following.

Lemma 2.3. There exist complex coefficients $a_{k}(p)$ and $\bar{a}_{k}(p)$, for all $k \in \overline{p-1} \cup\{0\}$, for all $p\left(\in \mathbf{Z}_{+}\right) \geq$ $\mu$, such that the formulas $A^{p}=\sum_{k=0}^{\mu-1} a_{k}(p) A^{k}=\sum_{k=0}^{p-1} \bar{a}_{k}(p) A^{k}$ are true. Those sets of coefficients coincide if $p=\mu$.

It turns out that he sets of expanding coefficients are real if the matrix $A$ is real or if it is complex being similar to a real one, that is, all complex values, if any, appear by complex conjugate pairs or identical multiplicities. Now, the above results are related to the fundamental matrices of time-invariant differential systems as follows. Consider $C_{0^{-}}$ semigroup $\left\{\Phi(t):=e^{A t}: t \in \mathbf{R}_{0+}\right\}$ of infinitesimal generator $A \in \mathbf{C}^{n \times n}$ is an evolution operator in $L\left(\mathbf{C}^{n}\right)$ point-wise defined by $\Phi(t)=e^{A t}$ the defining the trajectory solutions $x(t)=\Phi(t) x_{0}$ of the differential system of order $n \dot{x}(t)=A x(t) ; x(0)=x_{0}$. An equivalent description is that $\Phi: \mathbf{R}_{0+} \rightarrow \mathbf{C}^{n \times n}$ is the fundamental matrix function (or the state-transition matrix function) of the above differential system. The expansions of $\Phi(t)$ in finite powers of $A$, being not less than its minimal polynomial, where studied in detail for $A \in \mathrm{C}^{n \times n}$ in [4-7] by using Chebyshev sets of complex functions.

Lemma 2.4. The formulas $\Phi(t):=e^{A t}=\sum_{k=0}^{p-1} \beta_{k}(t, p) A^{k}$, for all $p \geq \mu$ for linearly independent sets of functions $B_{p}:=\left\{\beta_{k}: \mathbf{R}_{0+} \times \bar{p} \rightarrow \mathbf{R}: k \in \overline{p-1} \cup\{0\}\right\}$ which are unique and analytic in $\mathbf{R}_{+}$for each $p \geq \mu$ and which satisfy a $p$-order linear time-invariant differential system on $\mathbf{R}_{+}$.

Proof. It follows after using Lemma 2.3 with $A^{p}=\sum_{k=0}^{\mu-1} a_{k}(p) A^{k}=\sum_{k=0}^{p-1} \bar{a}_{k}(p) A^{k}$ that

$$
\begin{aligned}
\Phi(t) & :=e^{A t}=\sum_{k=0}^{\infty} \frac{A^{k} t^{k}}{k !}=\sum_{k=0}^{p-1} \frac{A^{k} t^{k}}{k !}+\sum_{k=p}^{\infty} \frac{A^{k} t^{k}}{k !}=\sum_{k=0}^{\mu-1} \frac{A^{k} t^{k}}{k !}+\sum_{k=\mu}^{\infty} \frac{A^{k} t^{k}}{k !} \\
& =\sum_{k=0}^{\mu-1}\left(\frac{t^{k}}{k !}+\sum_{i=\mu}^{\infty} \frac{a_{k}(i) t^{i}}{i !}\right) A^{k}=\sum_{k=0}^{\mu-1} \beta_{k}(t, \mu) A^{k} \\
& =\sum_{k=0}^{p-1}\left(\frac{t^{k}}{k !}+\sum_{i=p}^{\infty} \frac{\bar{a}_{k}(i) t^{i}}{i !}\right) A^{k}=\sum_{k=0}^{p-1} \beta_{k}(t, p) A^{k}, \quad \forall p \geq \mu
\end{aligned}
$$


with $\beta_{0}(0, p)=1, \beta_{i}(0, p)=0$; for all $i \in \overline{p-1} \cup\{0\}$, where

$$
\begin{gathered}
\beta_{k}(t, \mu)=\frac{t^{k}}{k !}+\sum_{i=\mu}^{\infty} \frac{a_{k}(i) t^{i}}{i !} ; \quad k \in \overline{\mu-1} \cup\{0\}, \\
\beta_{k}(t, p)=\frac{t^{k}}{k !}+\sum_{i=p}^{\infty} \frac{\bar{a}_{k}(i) t^{i}}{i !} ; \quad k \in \overline{p-1} \cup\{0\}, \forall p \geq \mu .
\end{gathered}
$$

Using the recursive expressions (2.9) for the $a_{(\cdot)}(\cdot)$ in the above formula and using also the recursions (2.12) for the $\bar{a}_{(\cdot)}(\cdot)$, for all $k \in \overline{p-1} \cup\{0\}$, one gets

$$
\begin{aligned}
& \beta_{k}(t, \mu)=\frac{t^{k}}{k !}+\sum_{i=\mu}^{\infty} \frac{t^{i}}{i !}\left(a_{k-\mu-1}^{i-k+\mu}(k-\mu) a_{k}(k-\mu)+\sum_{\ell=0}^{i-k+\mu-1} a_{k-\mu-1}^{\ell}(k-\mu) a_{k-1}(i-\ell-1)\right) \\
& k \in \overline{\mu-1} \cup\{0\}, a_{-1}(i)=0 ; \forall i \geq \mu, \\
& \beta_{k}(t, p)=\frac{t^{k}}{k !}+\sum_{i=p}^{\infty}\left(a_{k}(\mu)-\sum_{j=\mu}^{i-1} \bar{a}_{j}(i) a_{k}(k)\right) \frac{t^{i}}{i !} ; \quad k \in \overline{\mu-1} \cup\{0\}, \forall p \geq \mu .
\end{aligned}
$$

These functions satisfy sets of linear time-invariant ordinary differential equations as follows:

$$
\begin{aligned}
\dot{\Phi}(t) & =A \Phi(t)=\sum_{k=0}^{p-1} \dot{\beta}_{k}(t, p) A^{k}=\sum_{k=0}^{p-1} \beta_{k}(t, p) A^{k+1} \\
& =\sum_{k=1}^{p} \beta_{k-1}(t, p) A^{k}+\beta_{p-1}(t, p) A^{p} ; \quad p \geq \mu
\end{aligned}
$$

that implies

$$
\dot{\beta}_{0}(t, p) I_{n}+\sum_{k=1}^{p-1}\left(\dot{\beta}_{k}(t, p)-\beta_{k-1}(t, p)\right) A^{k}-\beta_{p-1}(t, p) A^{p}=0
$$

The above constraint holds for any matrix $A$ so that functions in the sets $\mathbf{B}_{p}:=\left\{\beta_{\ell}: \mathbf{R}_{0+} \times \bar{p} \rightarrow\right.$ $\mathbf{R}: \ell \in \overline{p-1} \cup\{0\}\}$, for all $p \geq \mu$ are linearly independent since they are the solutions of the linear time-invariant differential system

$$
\begin{gathered}
{\left[\begin{array}{c}
\dot{\beta}_{0}(t, p) \\
\dot{\beta}_{1}(t, p) \\
\vdots \\
\dot{\beta}_{p-1}(t, p)
\end{array}\right]=\left[\begin{array}{ccccc}
0 & 0 & \cdots & 0 & \bar{a}_{0}(p) \\
1 & 0 & \cdots & 0 & \bar{a}_{1}(p) \\
\ddots & \ddots & \vdots \\
0 & \cdots & 0 & 1 & \bar{a}_{p-1}(p)
\end{array}\right]\left[\begin{array}{c}
\beta_{0}(t, p) \\
\beta_{1}(t, p) \\
\vdots \\
\beta_{p-1}(t, p)
\end{array}\right],} \\
\beta_{0}(0, p)=1, \quad \beta_{i}(0, p)=0 ; \quad \forall i \in \overline{p-1} \cup\{0\}
\end{gathered}
$$


for all $p \geq \mu$,for all $k \in \overline{p-1} \cup\{0\}$, with $\bar{a}_{k}(\mu)=a_{k}(\mu)$, for all $k \in \overline{\mu-1} \cup\{0\}$, whose respective unique solutions are

$$
f_{\alpha}(t, p)=e^{\Omega_{a}(p) t} f_{\alpha}(0, p),
$$

where $f_{\beta}(t, p)=\left(\beta_{0}(t, p), \beta_{1}(t, p), \ldots, \beta_{p-1}(t, p)\right)^{T}$ with $f_{\beta}(0, p)=e_{1}=(1,0, \ldots, 0)^{T}$, and

$$
\Omega_{a}(p)=\left[\begin{array}{ccccc}
0 & 0 & \cdots & 0 & \bar{a}_{0}(p) \\
1 & 0 & \cdots & 0 & \bar{a}_{1}(p) \\
& \ddots & \ddots & \vdots \\
0 & \cdots & 0 & 1 & \bar{a}_{p-1}(p)
\end{array}\right] .
$$

Then, there exist unique sets $\mathbf{B}_{p}:=\left\{\beta_{k}: \mathbf{R}_{0+} \times \bar{p} \rightarrow \mathbf{R}: k \in \overline{p-1} \cup\{0\}\right\}$ of linearly independent functions, which are unique and analytic in $\mathbf{R}_{+}$for each $p \geq \mu$ from (2.19)-(2.20), such that the formulas $\Phi(t)=e^{A t}=\sum_{k=0}^{p-1} \beta_{k}(t, p) A^{k}$ hold, for all $t \in \mathbf{R}_{0+}$, for all $p \geq \mu$.

Remark 2.5. Note that it has been proven that there exist sets $\mathbf{A}_{p}:=\left\{a_{k}(p): k \in \overline{\mu-1} \cup\{0\}\right\}$ and $\overline{\mathbf{A}}_{p}:=\left\{\bar{a}_{k}(p): k \in \overline{p-1} \cup\{0\}\right\} ; p \geq \mu$ of complex coefficients, with $\bar{a}_{k}(\mu)=a_{k}(\mu)$, for all $k \in \overline{\mu-1} \cup\{0\}$, such that the following polynomial expansions are true $A^{\mu}=\sum_{k=0}^{\mu-1} a_{k}(\mu) A^{k}$, $A^{n}=\sum_{k=0}^{n-1} \bar{a}_{k}(n) A^{k}=\sum_{k=0}^{\mu-1} a_{k}(n) A^{k}$ where $\mu$ and $n$ are the degrees of the minimal and the characteristic polynomial of $A$, respectively. In general, $A^{p}=\sum_{k=0}^{\mu-1} a_{k}(\mu) A^{k}=\sum_{k=0}^{\mu-1} a_{k}(p) A^{k}=$ $\sum_{k=0}^{p-1} \bar{a}_{k}(p) A^{k}$ for $p \geq \mu$. Those sets are unique for each $p \geq \mu$. The above formulas imply that there exist unique sets $\mathbf{B}_{p}:=\left\{\beta_{k}: \mathbf{R}_{0+} \times \bar{p} \rightarrow \mathbf{R}: k \in \overline{p-1} \cup\{0\}\right\}$ of linearly independent functions, which are unique and analytic in $\mathbf{R}_{+}$for each $p \geq \mu$ from (2.19)-(2.20), such that the formulas $\Phi(t)=e^{A t}=\sum_{k=0}^{p-1} \beta_{k}(t, p) A^{k}$ hold, for all $p \geq \mu$.

The following result is useful to relate the coefficients of the minimal and characteristic polynomials of $A$ to the two more relevant versions of the Cayley-Hamilton theorem.

Lemma 2.6. Assume that $p_{n}(A)=\operatorname{det}\left(s I_{n}-A\right)=\operatorname{det}\left(s I_{n}-J_{A}\right)$ is the characteristic polynomial of a nonzero matrix $A$ of $n$ order and Jordan form $J_{A}$. Then, $p_{n}(A)=s^{n}-\sum_{k=0}^{n-1} \bar{a}_{k}(n) s^{k}$.

Assume that $\mu$, with $1 \leq \mu \leq n$, is the degree of the minimal polynomial of $A, p_{\mu}(A)$. Then, $p_{\mu}(A)=p_{\mu}\left(J_{A}\right)=p_{\mu}\left(\widehat{J}_{A}\right)=s^{\mu}-\sum_{k=0}^{\mu-1} a_{k}(\mu) s^{k}$, where $\widehat{J}_{A}=E^{T} J_{A} E=E^{T} T^{-1} J_{A} T E$ is a $\mu$-square matrix and $T$ and $E$ are $n$-square nonsingular and $\mu \times n$ matrices, respectively, which are unique except multiplication by a nonzero scalar and $p_{\mu}(A)$ is the characteristic and minimal polynomial of $\widehat{J}_{A}$.

Proof. The first part follows from the Cayley-Hamilton theorem in the form $A^{n}=$ $\sum_{k=0}^{n-1} \bar{a}_{k}(n) A^{k}$ and the fact that the matrix satisfies its own characteristic polynomial. If $\mu$ is the degree of the minimal polynomial of $A$, then there exist a nonsingular real $n$-matrix $T$ and a $\mu \times n$ real matrix $E$, formed by identity and zero-block matrices of appropriate orders which pickup from $J_{A}$ the higher-order Jordan blocks of each eigenvalue of $A$, whose orders are given by the respective index of each eigenvalue, such that the identities 
$\widehat{J}_{A}=E^{T} J_{A} E=E^{T} T^{-1} J_{A} T E$ hold. The last identity holds from the Cayley-Hamilton theorem since the characteristic polynomial of $\widehat{J}_{A}$ and the minimal polynomial of $A$ and $J_{A}$ coincide.

\section{The Expansions of Evolution Operators of Functional Fractional Caputo Differential Systems and Their Associate Chebyshev Systems}

The above results are extended to the following fractional Caputo differential systems of order $\alpha$ :

$$
\left({ }^{C} D_{0+}^{\alpha} x\right)(t)=A x(t)+B u(t),
$$

with $k-1<\alpha\left(\in \mathbf{R}_{+}\right) \leq k$, for some $k-1, k \in \mathbf{Z}_{0_{+}}$and $B \in \mathbf{R}^{n \times m}$ is the control matrix. If $\alpha=1$, then (3.1) is referred to in the sequel as the standard system. The initial conditions are $\varphi_{j}(0)=$ $x_{j}(0)=x^{(j)}(0)=x_{0 j}$, for all $j \in \overline{k-1} \cup\{0\}$. The admissible function vector $u: \mathbf{R}_{0+} \rightarrow \mathbf{R}^{m}$ is any given bounded piecewise continuous control function. The following result is concerned with the unique solution on $\mathbf{R}_{0+}$ of the above differential fractional system (3.1). The proof follows directly from a parallel existing result from the background literature on fractional differential systems by grouping all the additive forcing terms of (3.1) in a unique one (see, e.g., [1], equations (1.8.17), (3.1.34)- (3.1.49), with $f(t) \equiv A x(t)+B u(t))$.The linear and timeinvariant differential functional fractional differential system (3.1) of any order $\alpha \in \mathbf{C}_{0+}$ has the following unique solution on $\mathbf{R}_{0+}$ for each given set of initial conditions and each given control $u: \mathbf{R}_{0+} \rightarrow \mathbf{R}^{m}$ being a bounded piecewise continuous control function, [1]:

$$
x_{\alpha}(t)=\sum_{j=0}^{k-1} t^{j} \Phi_{\alpha j}(t) x_{0 j}+\int_{0}^{t}(t-\tau)^{\alpha-1} \Phi_{\alpha}(t-\tau) B u(\tau) d \tau ; \quad t \in \mathbf{R}_{0+}
$$

with $k=[\operatorname{Re} \alpha]+1$ if $\alpha \notin \mathbf{Z}_{+}$and $k=\alpha$ if $\alpha \in \mathbf{Z}_{+}$. Direct calculations yield that for any set of initial conditions the solution (3.2) verifies (3.1). Uniqueness is direct from PicardLindeloff theorem. The matrix functions $\Phi_{\alpha j}(t)$, for all $j \in \overline{k-1} \cup\{0\}$ and $\Phi_{\alpha}(t)$ from $\mathbf{R}_{0+}$ to $\mathbf{R}^{n \times n}$ are calculated via the Mittag-Leffler functions which, after using the identities $A^{\ell}=$ $\sum_{\ell=0}^{\mu-1} a_{\ell}(\ell) A^{\ell}=\sum_{\ell=0}^{p-1} \bar{a}_{\ell}(\ell) A^{\ell}$, for all $p \geq \mu$ from Lemma 2.3 for the matrix $A$, lead to

$$
\begin{aligned}
\Phi_{\alpha j}(t) & =\sum_{\ell=0}^{\infty} \frac{A^{\ell} t^{\alpha \ell}}{\Gamma(\alpha \ell+j+1)}=\sum_{\ell=0}^{\mu-1}\left(\frac{t^{\alpha \ell}}{\Gamma(\alpha \ell+j+1)}+\sum_{i=\mu}^{\infty} \frac{\bar{a}_{\ell}(i) t^{\alpha i}}{\Gamma(\alpha i+j+1)}\right) A^{\ell} \\
& =\sum_{\ell=0}^{\mu-1} \beta_{\alpha j \ell}(t, \mu) A^{\ell} ; \quad \forall j \in \overline{k-1} \cup\{0\} \\
& =\sum_{\ell=0}^{p-1}\left(\frac{t^{\alpha \ell}}{\Gamma(\alpha \ell+j+1)}+\sum_{i=p}^{\infty} \frac{\bar{a}_{\ell}(i) t^{\alpha i}}{\Gamma(\alpha i+j+1)}\right) A^{\ell}
\end{aligned}
$$




$$
\begin{aligned}
& =\sum_{\ell=0}^{p-1} \beta_{\alpha j \ell}(t, p) A^{\ell} ; \quad \forall p \geq \mu, \forall j \in \overline{k-1} \cup\{0\} \\
\Phi_{\alpha}(t) & =\sum_{\ell=0}^{\infty} \frac{A^{\ell} t^{\alpha \ell}}{\Gamma((\ell+1) \alpha)}=\sum_{\ell=0}^{p-1} \frac{A^{\ell} t^{\alpha \ell}}{\Gamma((\ell+1) \alpha)}+\sum_{\ell=p}^{\infty} \frac{A^{\ell} t^{\alpha \ell}}{\Gamma((\ell+1) \alpha)} \\
& =\sum_{\ell=0}^{p-1}\left(\frac{t^{\alpha \ell}}{\Gamma((\ell+1) \alpha)}+\sum_{i=p}^{\infty} \frac{\bar{a}_{k}(\ell) t^{\alpha i}}{\Gamma((i+1) \alpha)}\right) A^{\ell} ; \quad \forall p \geq \mu \\
& =\sum_{\ell=0}^{p-1} \beta_{\alpha \ell}(t, p) A^{\ell} ; \quad \forall p \geq \mu,
\end{aligned}
$$

for all $t \in \mathbf{R}_{0+}$, where, provided that $k-1<\alpha\left(\in \mathbf{R}_{+}\right) \leq k\left(\in \mathbf{Z}_{+}\right), \Gamma: \mathbf{R}_{0+} \rightarrow \mathbf{R}_{+}$is the $\Gamma$-function of definition domain restricted to $\mathbf{R}_{0+}$ and the elements of the sets of functions $B_{\alpha j p}:=\left\{\beta_{\alpha j \ell}: \mathbf{R}_{0+} \times \bar{p} \rightarrow \mathbf{R}: \ell \in \overline{p-1} \cup\{0\}\right\}$, for all $j \in \overline{k-1} \cup\{0\}$ and $B_{\alpha p}:=\left\{\beta_{\alpha \ell}: \mathbf{R}_{0+} \times \bar{p} \rightarrow\right.$ $\mathbf{R}: \ell \in \overline{p-1} \cup\{0\}\}$, for all $p \geq \mu$ are defined for $t \in \mathbf{R}_{0+}$ as follows:

$$
\begin{gathered}
\beta_{\alpha j \ell}(t, p)=\left(\frac{t^{\alpha \ell}}{\Gamma(\alpha \ell+j+1)}+\sum_{i=p}^{\infty} \frac{\bar{a}_{\ell}(i) t^{\alpha i}}{\Gamma(\alpha i+j+1)}\right) ; \quad \forall j \in \overline{k-1}, \forall \ell \in \overline{p-1} \cup\{0\}, \forall p \geq \mu, \\
\beta_{\alpha \ell}(t, p)=\left(\frac{t^{\alpha \ell}}{\Gamma((\ell+1) \alpha)}+\sum_{i=p}^{\infty} \frac{\bar{a}_{k}(\ell) t^{\alpha i}}{\Gamma((i+1) \alpha)}\right) ; \quad \forall p \geq \mu .
\end{gathered}
$$

Remark 3.1. Note that the homogeneous solution of the fractional differential system is given by

$$
x_{\alpha}(t)=\sum_{j=0}^{k-1} t^{j} \Phi_{\alpha j}(t) x_{0 j}
$$

Then,

$$
\left({ }^{C} D_{0+}^{\alpha}\left(\sum_{j=0}^{k-1} t^{j} \Phi_{\alpha j}(t)\right)\right)(t)=\sum_{j=0}^{k-1}\left(A t^{j} \Phi_{\alpha j}(t)\right)
$$

(see, e.g., $[1,3])$. On the other hand, if only one of the above point initial conditions $\varphi_{j}(0)=$ $x_{j}(0)=x^{(j)}(0)=x_{0 j}$ is nonzero for some arbitrary $j \in \overline{k-1} \cup\{0\}$, then (3.7) is decomposable for each additive term resulting in:

$$
x_{\alpha}(t)=t^{j} \Phi_{\alpha j}(t) x_{0 j} .
$$


Thus, $\Phi_{\alpha j}(t)$ is a fundamental matrix for all $j \in \overline{k-1} \cup\{0\}$ which satisfies the fractional differential system of order $\alpha$

$$
\left({ }^{C} D_{0+}^{\alpha} t^{j} \Phi_{\alpha j}\right)(t)=A t^{j} \Phi_{\alpha j}(t)=\left({ }^{C} D_{0+}^{\alpha}\left(\sum_{\ell=0}^{p-1} t^{j} \beta_{\alpha j \ell}(t, p) A^{\ell}\right)\right)(t)=\left(\sum_{\ell=0}^{p-1} t^{j} \beta_{\alpha j \ell}(t, p) A^{\ell+1}\right),
$$

for all $j \in \overline{k-1} \cup\{0\}$. Note that the fractional system becomes the standard one for $j=0$ and $\alpha=1$ so that $\beta_{10 p}=\beta_{p}$ (defined in Lemma 2.4) and $\Phi(t)=e^{A t}$.

The following result of Section 2 extends the linear independence of the functions expanding $e^{A t}$ to those appearing in the expansions of $\Phi_{\alpha j}(t)$, for all $j \in \overline{k-1} \cup\{0\}$ and $\Phi_{\alpha}(t)$

Lemma 3.2. All the sets of functions $B_{\alpha j p}:=\left\{\beta_{\alpha j \ell}: \mathbf{R}_{0+} \times \bar{p} \rightarrow \mathbf{R}: \ell \in \overline{p-1} \cup\{0\}\right\}$ and $B_{\alpha p}:=\left\{\beta_{\alpha \ell}: \mathbf{R}_{0+} \times \bar{p} \rightarrow \mathbf{R}: \ell \in \overline{p-1} \cup\{0\}\right\}$, for all $p\left(\in \mathbf{Z}_{+}\right) \geq \mu$ are analytic and linearly independent on $\mathbf{R}_{+}$for any given $\alpha \in \mathbf{R}_{+}, k \in \mathbf{Z}_{+}$fulfilling $k-1<\alpha \leq k$ and for all $j \in \overline{k-1} \cup\{0\}$, for all $p\left(\in \mathbf{Z}_{+}\right) \geq \mu$. Furthermore, the sets $B_{\alpha j p}$ satisfy a p-order linear time-invariant fractional differential system on $\mathbf{R}_{+}$, for all $j \in \overline{k-1} \cup\{0\}$.

Proof. The sets of functions are analytic since from their defining formulas, it follows that they are infinitely differentiable on $\mathbf{R}_{+}$. Their linear independence is proven by contradiction. Since the rows of the fundamental matrices of solutions $\Phi_{\alpha j}(t)$; for all $j \in \overline{k-1} \cup\{0\}$ of the Caputo fractional differential system of order $\alpha$ are linearly independent on $\mathbf{R}_{0+}$, it follows that $\Phi_{\alpha j}^{T}(t) \lambda=0 \Leftrightarrow \lambda=0$ for any $\lambda:=\left(\lambda_{1}, \lambda_{2}, \ldots, \lambda_{p}\right)^{T} \in \mathbf{R}^{p}$; for all $p \geq \mu$. But, if $B_{\alpha j p}$ is a linearly dependent set on $\mathbf{R}_{0+}$, since $\left[\begin{array}{llll}I_{n} & A^{T} & \cdots & A^{p-1^{T}}\end{array}\right]$ is a full row rank matrix, then it exists $\lambda \neq 0$ such that

$$
t^{-j} \Phi_{\alpha j}^{T}(t) \mathcal{\lambda}=\left[\begin{array}{llll}
I_{n} & A^{T} & \cdots & A^{p-1^{T}}
\end{array}\right]\left[\begin{array}{c}
\beta_{\alpha j 0}(t, p) I_{n} \\
\beta_{\alpha j 1}(t, p) I_{n} \\
\vdots \\
\beta_{\alpha j, p-1}(t, p) I_{n}
\end{array}\right] \lambda=0 \Longleftrightarrow\left[\begin{array}{c}
\beta_{\alpha j 0}(t, p) I_{n} \\
\beta_{\alpha j 1}(t, p) I_{n} \\
\vdots \\
\beta_{\alpha j, p-1}(t, p) I_{n}
\end{array}\right] \lambda=0,
$$

for all $t \in \mathbf{R}_{+}$which contradicts that $\lambda=0$ in order that $\Phi_{\alpha j}(t)$ be a fundamental matrix, that is, their rows are linearly independent functions. The functions in the set $B_{\alpha p}:=\left\{\beta_{\alpha \ell}\right.$ : $\left.\mathbf{R}_{0+} \times \bar{p} \rightarrow \mathbf{R}: \ell \in \overline{p-1} \cup\{0\}\right\}$ are also linearly independent from the above considerations for the particular case $\alpha=k, j=k-1$ of $B_{\alpha j p}$. Note that the functions in the various sets are zero for $t=0$ so that linear independence is restricted to $\mathbf{R}_{+}$. On the other hand, the functions in the set $B_{\alpha j p}$, satisfy the differential system

$$
\left({ }^{C} D_{0+}^{\alpha}\left(\sum_{\ell=0}^{p-1} \beta_{\alpha j \ell}(t, p) A^{\ell}\right)\right)(t)=\left(\sum_{\ell=0}^{p-1} \beta_{\alpha j \ell}(t, p) A^{\ell+1}\right),
$$


for all $j \in \overline{k-1} \cup\{0\}$, subject to initial conditions $\beta_{\alpha j 0}(0, p)=1 / \Gamma(j+1)=1 / j !, \beta_{\alpha j \ell}(0, p)=0$, for all $j \in \overline{k-1}$, for all $\ell \in \overline{p-1}$, for all $p \geq \mu$. The functions in the sets $B_{\alpha p}$ satisfy initial conditions $\beta_{\alpha 0}(0, p)=1 / \Gamma(\alpha+1)=1 /(\alpha \Gamma(\alpha))$ for all $j \in \overline{k-1}, \beta_{\alpha j \ell}(0, p)=\beta_{\alpha \ell}(0, p)=0$, for all $j \in \overline{k-1}$, for all $\ell \in \overline{p-1}$, for all $p \geq \mu$.

Remark 3.3. Note that, in the nonfractional standard case, the solution is also obtained from the fractional solution of order $\alpha$ (3.2) as a particular case for $\alpha=1, j=0, k=1$ which results to yield $\Phi_{10}(t)=\Phi_{1}(t)=e^{A t}$, for all $t \in \mathbf{R}_{0+}$, that is, the fundamental matrix appears simultaneously for the homogeneous solution and for the zero-initial-state forced one and in such a case is the exponential matrix function which also defines a $C_{0}$-semigroup of infinitesimal generator $A$. It can be pointed out that in the fractional case $\alpha \neq 1$, the fundamental matrix is not an exponential matrix function as it follows from (3.3). The linear independence of functions in Lemma 3.2 applies for the general fractional case as for the nonfractional case. This is an essential point for the non-uniform sampling case discussed later on in this paper.

\section{Observability and Controllability of Linear Fractional Differential Time-Invariant Systems}

Consider the Caputo fractional differential system (3.1) with a measurable output defined by

$$
y_{\alpha}(t)=C x_{\alpha}(t)
$$

for some $C \in \mathbf{R}^{z \times n}$ with $z\left(\in \mathbf{Z}_{+}\right) \leq n$. The following observability property is characterized.

Definition 4.1. The Caputo fractional differential system of order $\alpha$ is said to be observable in the observation time interval $[0, t]$ if $x_{j}(0)=x_{0 j}$, for all $j \in \overline{k-1} \cup\{0\}$ can be uniquely calculated from the measurable output $y_{\alpha}(t) ; t \in[0, t]$ for some real interval $[0, t]$ of nonzero measure.

Theorem 4.2. The Caputo fractional differential system (3.1), (4.1) of order $\alpha$ is observable in $[0, t]$ for any finite $t \in \mathbf{R}_{+}$only if the standard dynamic system, that is, that resulting when $\alpha=1$, that is,

$$
\dot{x}(t)=A x(t)+B u(t) ; \quad y(t)=C x(t)
$$

is observable.

Proof. First, consider the homogeneous Caputo fractional differential system (3.1), that is, $u \equiv 0$. One gets from (3.2)-(3.3) that

$$
\begin{aligned}
y_{\alpha}(t) & =\left(\sum_{j=0}^{k-1} t^{j} C \Phi_{\alpha j}(t) x_{0 j}\right)=\left(\sum_{j=0}^{k-1} \sum_{\ell=0}^{p-1} t^{j} C \beta_{\alpha j \ell}(t, p) A^{\ell} x_{0 j}\right) \\
& =\bar{\beta}_{\alpha}^{T}(t, p) \text { Block Diag }[\operatorname{Ob}(A, C, p): \underbrace{k}_{\cdots} \vdots \mathbf{O b}(A, C, p)] \bar{x}_{0},
\end{aligned}
$$


where $\bar{x}_{0}:=\left[x_{00}^{T}, x_{01}^{T}, \ldots, x_{0, k-1}^{T}\right]^{T}$, and

$$
\bar{\beta}_{\alpha}^{T}(t, p):=\left[\begin{array}{lllllll}
\beta_{\alpha 00}(t, p) I_{s} & \cdots & \beta_{\alpha 0, p-1}(t, p) I_{S} & \cdots & t^{k-1} \beta_{\alpha, k-1,0}(t, p) I_{s} & \cdots & t^{k-1} \beta_{\alpha, k-1, p-1}(t, p) I_{s}
\end{array}\right]
$$

for all $t\left(\in \mathbf{R}_{0+}\right)$ is a $s \times p k s$ real matrix function; for all $p \geq \mu$ where $\operatorname{Ob}(A, C, \mu):=\left[\begin{array}{c}C \\ C A \\ C A^{\mu-1}\end{array}\right]$. Since (4.2) is observable, then

$$
\begin{gathered}
\operatorname{rank} \text { Block Diag }[\mathrm{Ob}(A, C, p): \underbrace{k}_{\cdots}: \mathbf{O b}(A, C, p)] \\
=k n \Longleftrightarrow \operatorname{rank} \mathrm{Ob}(A, C, \mu)=\operatorname{rank} \mathbf{O b}(A, C, p)=n .
\end{gathered}
$$

Furthermore, since the sets $B_{\alpha j p}:=\left\{\beta_{\alpha j \ell}: \mathbf{R}_{0+} \times \bar{p} \rightarrow \mathbf{R}: \ell \in \overline{p-1} \cup\{0\}\right\}$ are linearly independent on $\mathbf{R}_{+}$from Lemma 3.2, the map from $\mathbf{R}^{k n}$ to $\mathbf{R}^{s} \times[0, t]$, for all $t \in \mathbf{R}_{+}$from the initial conditions to the output-trajectory of the homogeneous system defined by (4.3) is injective. Thus, $\bar{x}_{0}$ can be uniquely calculated from $y: \mathbf{R}^{k n} \rightarrow \mathbf{R}^{s} \times[0, t]$. The sufficiency part has been proven. The necessity is obvious since if the rank in (4.4) is less than $n$, so that (4.2) is not observable, then the above mentioned map from $\mathbf{R}^{k n}$ to $\mathbf{R}^{s} \times[0, t]$ is not injective. The above considerations on (4.3)-(4.5) may be extended directly to the case that the admissible control $u(t)$ is nonzero with its proof remaining valid, by replacing via (3.2) and (4.1)

$$
y_{\alpha}(t)=C x_{\alpha}(t) \longrightarrow \bar{y}_{\alpha}(t)=y(t)-\int_{0}^{t}(t-\tau)^{\alpha-1} C \Phi_{\alpha}(t-\tau) B u(\tau) d \tau
$$

Remark 4.3. Note that Definition 4.1 and Theorem 4.2 characterize the observability property on any time interval of nonzero measure so that the property is independent of the time interval used for observation purposes. On the other hand, the property is independent of $\alpha$ because it implies the observability of the standard system (4.2) and it is implied by such an observability.

Remark 4.3 is formally enounced in the subsequent result.

Corollary 4.4. The observability property of the Caputo fractional differential system (3.1), (4.1) is independent of any real fractional order $\alpha$, with $k-1<\alpha \leq k$ for any given $k \in \mathbf{Z}_{+}$, and independent of the observation interval provided it is of nonzero measure.

The observability property can be tested as follows. 
Corollary 4.5. The Caputo fractional differential system (3.1), (4.1) of any real order $\alpha$, with $k-1<$ $\alpha \leq k$ for any given $k \in \mathbf{Z}_{+}$, is observable if and only if any of the three equivalent conditions holds

(1)

$$
\operatorname{rank}\left[C^{T}: A^{T} C^{T}: \ldots \vdots A^{T \mu-1} C^{T}\right]=n,
$$

(2)

$$
\operatorname{rank}\left[\lambda I_{n}-A^{T}: C^{T}\right]=n ; \quad \forall \lambda \in \sigma(A)
$$

where $\sigma(A)$ is the spectrum of $A$,

(3) $\int_{0}^{t} \Psi_{\alpha}^{T}(\tau) C^{T} C \Psi_{\alpha}(\tau) d \tau>0$, for any finite time interval $[0, t]$ of nonzero measure

$$
\Psi_{\alpha}(t):=\left[\Phi_{\alpha 0}(t): t \Phi_{\alpha 1}(t) \vdots \cdots: t^{k-1} \Phi_{\alpha, k-1}(t)\right]
$$

Proof. The equivalence of Conditions (1)-(2) follows from the equivalence of the observability test for the standard system (i.e., $\alpha=k=1$ ) with the Popov-Belevitch-Hautus spectral observability test, $[8,17]$, with the observability of any Caputo fractional system of order $\alpha$ with $k-1<\alpha \leq k$ by using Corollary 4.4. The equivalence of Conditions (1)-(2) with Condition 3 follows from the fact that the observability of the fractional system of order $\alpha$ is uniform with respect to time (Theorem 4.2 or Corollary 4.4) and the fact that (3.6) into (4.1) with (4.9) yields for the unique (nontrivial) measurable output trajectory of the homogeneous fractional system of order $\alpha$ for each $\bar{x}_{0}(\neq 0) \in \mathbf{R}^{n k}$

$$
0<\int_{0}^{t} y_{\alpha}^{T}(\tau) y_{\alpha}(\tau) d \tau=\bar{x}_{0}^{T}\left(\int_{0}^{t} \Psi_{\alpha}^{T}(\tau) C^{T} C \Psi_{\alpha}(\tau) d \tau\right) \bar{x}_{0}
$$

for any time interval $[0, t]$ finite of nonzero measure. Thus, $\bar{x}_{0}(\neq 0) \in \mathbf{R}^{n k}$ is unique from (4.10), and then the system is observable, if and only if $\int_{0}^{t} \Psi_{\alpha}^{T}(\tau) C^{T} C \Psi_{\alpha}(\tau) d \tau>0$. Thus, Condition (3) is equivalent to Conditions (1)-(2).

Remark 4.6. Note from Condition (3) of Corollary 4.5 that if the Caputo fractional system of order $\alpha$, with $k-1<\alpha \leq k$, is observable, then

$$
\int_{0}^{t} \tau^{2 j} \Phi_{\alpha j}^{T}(\tau) C^{T} C \Phi_{\alpha j}(\tau) d \tau>0 \Longleftrightarrow \int_{0}^{t} \Phi_{\alpha j}^{T}(\tau) C^{T} C \Phi_{\alpha j}(\tau) d \tau>0 ; \quad \forall j \in \overline{k-1} \cup\{0\},
$$

for any finite time interval $[0, t]$ of nonzero measure. This is easily seen by taking initial conditions such that $x_{0 j} \neq 0$ for some $j \in \overline{k-1} \cup\{0\}$ while $x_{0 i}=0$, for all $i(\neq j) \in \overline{k-1} \cup\{0\}$. 
Remark 4.7. Note that Theorem 4.2 is alternatively proven from the spectral observability test as follows. Since the rank in (4.8) can only be lost for $\lambda \in \sigma(A)$, then (4.8) is equivalent to

$$
\operatorname{rank}\left[\lambda I_{n}-A^{T}: C^{T}\right]=n ; \quad \forall \lambda \in \mathbf{C}
$$

Using Laplace transform of (3.1), [2], the spectral observability of the Caputo fractional system of order $\alpha$ is lost if and only if $\operatorname{rank}\left[\lambda^{\alpha} I_{n}-A^{T}: C^{T}\right]<n$ for some $\lambda \in \mathrm{C}$. Thus, note that

$$
\begin{gathered}
\operatorname{rank}\left[\lambda I_{n}-A^{T}: C^{T}\right]<n, \quad \text { some } \lambda \in \mathbf{C} \Longrightarrow \operatorname{rank}\left[\lambda_{1}^{\alpha} I_{n}-A^{T}: C^{T}\right]<n \quad \text { for } \lambda_{1}=\lambda^{1 / \alpha}, \\
\operatorname{rank}\left[\lambda^{\alpha} I_{n}-A^{T}: C^{T}\right]<n, \quad \text { some } \lambda \in \mathbf{C} \Longrightarrow \operatorname{rank}\left[\lambda_{1} I_{n}-A^{T}: C^{T}\right]<n \quad \text { for } \lambda_{1}=\lambda^{\alpha} .
\end{gathered}
$$

Then, the Caputo fractional system of order $\alpha$, with $k-1<\alpha \leq k$, is (is not) spectrally observable (equivalently, observable) if and only if the standard system $\alpha=k=1$ is (is not) spectrally observable.

The controllability property is now discussed.

Definition 4.8. The Caputo fractional differential system of order $\alpha$ is said to be controllable in the time interval $[0, t]$ of nonzero finite measure if it exists an admissible control $u:[0, t] \rightarrow$ $\mathbf{R}^{m}$ which steers the state-trajectory solution to any prescribed value $x^{*}=x_{\alpha}(t)$ for any given initial conditions.

Theorem 4.9. The Caputo fractional differential system (3.1), (4.1) of any real order $\alpha$, with $k-1<$ $\alpha \leq k$ for any given $k \in \mathbf{Z}_{+}$, is controllable if and only if any of the three equivalent conditions holds:

(1)

$$
\operatorname{rank}\left[B: A B: \cdots \vdots A^{\mu-1} B\right]=n
$$

(2)

$$
\operatorname{rank}\left[\lambda I_{n}-A \vdots B\right]=n ; \quad \forall \lambda \in \sigma(A)
$$

where $\sigma(A)$ is the spectrum of $A$,

(3) $\int_{0}^{t} \Phi_{\alpha}(\tau) B B^{T} \Phi_{\alpha}(\tau) d \tau>0$, for any finite time interval $[0, t]$ of nonzero measure.

Proof. Assume the forced solution of (3.2) under zero initial conditions, namely, $x_{j}(0)=x_{j 0}=$ 0 ; for all $j \in \overline{k-1} \cup\{0\}$ so that 


$$
\begin{aligned}
x_{\alpha}(t)= & \int_{0}^{t}(t-\tau)^{\alpha-1} \Phi_{\alpha}(t-\tau) B u(\tau) d \tau=\sum_{\ell=0}^{p-1}\left(\int_{0}^{t}(t-\tau)^{\alpha-1} \beta_{\alpha \ell}(t-\tau, p) A^{\ell} B u(\tau) d \tau\right) \\
= & \left(\int_{0}^{t}(t-\tau)^{\alpha-1}\left[\beta_{\alpha 0}(t-\tau, p), \beta_{\alpha 1}(t-\tau, p), \ldots, \beta_{\alpha, p-1}(t-\tau, p)\right] u(\tau) d \tau\right) \\
& \times\left[B^{T}: B A^{T}: \ldots B^{T} A^{p-1^{T}}\right]^{T} \\
= & \operatorname{Co}(A, B, p) \bar{\gamma}_{u \alpha}(t, p),
\end{aligned}
$$

for all $t \in \mathbf{R}_{0+}$, for all $p \geq \mu$ after using (3.4) where $\boldsymbol{\gamma}_{u \alpha i}(t, p):=\int_{0}^{t}(t-\tau)^{\alpha-1} \beta_{\alpha i}(t-\tau, p) u(\tau) d \tau$ are $m$-vector functions being dependent on the control; for all $i \in \overline{p-1} \cup\{0\}$; for all $t \in \mathbf{R}_{0+}$, and

$$
\begin{gathered}
\operatorname{Co}(A, B, p):=\left[B \vdots A B \vdots \ldots \vdots A^{p-1} B\right] \\
\bar{\gamma}_{u \alpha}(t, p):=\left[r_{u \alpha 0}^{T}(t, p), r_{u \alpha 1}^{T}(t, p), \ldots, r_{u \alpha, p-1}^{T}(t, p)\right]^{T} ; \quad \forall t \in \mathbf{R}_{0+}
\end{gathered}
$$

are, respectively, a $n \times p m$ real matrix and a real $p m$-vector function. Since the functions in $\beta_{\alpha p}$ are linearly independent and analytic on $\mathbf{R}_{+}$, for all $p\left(\in \mathbf{Z}_{+}\right) \geq \mu$ for any given $\alpha \in \mathbf{R}_{+}$ (with $k-1<\alpha \leq k$-Lemma 3.2), then $Y_{\text {uap }}:=\left\{\boldsymbol{\gamma}_{\text {uai }}: \mathbf{R}_{0+} \times \bar{p} \rightarrow \mathbf{R}: i \in \overline{p-1} \cup\{0\}\right\}$, for all $p\left(\in \mathbf{Z}_{+}\right) \geq \mu$ is, by construction, a linearly independent set of analytic $m$-vector functions on a finite nonzero measure time interval $[0, t] \subset \mathbf{R}_{+}$for any given $\alpha \in \mathbf{R}_{+}, k-1<\alpha \leq k$ provided that the control is nonzero on some subset of nonzero measure of $[0, t]$. Thus, note from (4.17) that for arbitrary $\mathbf{R}^{n} \ni x^{*}=x_{\alpha}(t)$ for some linearly independent set $Y_{u \alpha p}$ on $[0, t]$ defined for some admissible nonzero control $u:[0, t] \rightarrow \mathbf{R}^{m}$ the Caputo fractional differential system of order $\alpha$ is controllable, independent of $\alpha$, if and only if

$$
\operatorname{rank}\left[\operatorname{Co}(A, B, \mu) \vdots x^{*}\right]=\operatorname{rank} \operatorname{Co}(A, B, p)=\operatorname{rank} \operatorname{Co}(A, B, \mu)=n
$$

what follows from Rouché-Froebenius theorem from Linear Algebra. Then, the controllability is guaranteed by that of the standard system with $\alpha=k=1$. The equivalence of (4.15) with the spectral controllability condition (4.16) follows from Theorem 4.2 and the well-known duality result and the state trajectory solution (3.2). The pair $(A, C)$ is observable in the sense that (4.7), equivalently (4.8), holds if its dual pair $\left(A^{T}, C^{T}\right)$ is controllable. The equivalence of Conditions (1)-(2) with Condition (3) is proven as follows from (4.16). Assume that the control is generated as $u(\tau)=B^{T} \Phi_{\alpha}(t-\tau) K ; \tau \in[0, t]$ for some $K \in \mathbf{R}^{m}$. Then, the controllability constraint

$$
x^{*}=x_{\alpha}(t)=\left(\int_{0}^{t}(t-\tau)^{\alpha-1} \Phi_{\alpha}(t-\tau) B B^{T} \Phi_{\alpha}(t-\tau) d \tau\right) K
$$


is solvable for any prescribed $x^{*} \in \mathbf{R}^{n}$ if and only if

$$
\int_{0}^{t}(t-\tau)^{\alpha-1} \Phi_{\alpha}(t-\tau) B B^{T} \Phi_{\alpha}(t-\tau) d \tau>0 \Longleftrightarrow \int_{0}^{t} \Phi_{\alpha}(t-\tau) B B^{T} \Phi_{\alpha}(t-\tau) d \tau>0
$$

with the control solution being

$$
u(\tau)=B^{T} \Phi_{\alpha}(t-\tau)\left(\int_{0}^{t}(t-\tau)^{\alpha-1} \Phi_{\alpha}(t-\tau) B B^{T} \Phi_{\alpha}(t-\tau) d \tau\right)^{-1} x^{*} ; \quad \tau \in[0, t] .
$$

Then, the Caputo fractional differential system of any order $\alpha$ is controllable independent of $\alpha$ on $[0, t]$ if $\int_{0}^{t} \Phi_{\alpha}(t-\tau) B B^{T} \Phi_{\alpha}(t-\tau) d \tau>0$. Sufficiency has been proven. Necessity follows by contradiction. Assume that the system is controllable and $\int_{0}^{t} \Phi_{\alpha}(t-\tau) B B^{T} \Phi_{\alpha}(t-\tau) d \tau>0$ fails for a given $[0, t]$ of nonzero finite measure. Then, the columns of the matrix function $\Phi_{\alpha}(t-\tau) B u(\tau)$ are not linearly independent vector functions on $[0, t]$ for any admissible control $u:[0, t] \rightarrow \mathbf{R}^{m}$. Then, from (4.17)-(4.18a), (4.18b) either $\operatorname{rank}\left[B \vdots A B \vdots \cdots \vdots A^{\mu-1} B\right]<n$ which contradicts the controllability Condition (1) or the set $Y_{\text {uap }}$ is not linearly independent on $[0, t]$ for any admissible control what is impossible if $\operatorname{rank}\left[B \vdots A B: \ldots \vdots A^{\mu-1} B\right]=n$. Then, Condition (3) is equivalent to the equivalent Conditions (1)-(3). All the proof may be easily readdressed for nonzero initial conditions by replacing

$$
x^{*}=x_{\alpha}(t) \longrightarrow x^{*}=\left(x_{\alpha}(t)-\sum_{j=0}^{k-1} t^{j} \Phi_{\alpha j}(t) x_{0 j}\right) \text {. }
$$

Note that, although the controllability property is independent of $\alpha$, the control (4.21) depends on $\alpha$. The matrices $\operatorname{Co}(A, B, \mu):=\left[B: A B: \cdots: A^{\mu-1} B\right]$ and $\operatorname{Ob}(A, C, \mu):=\left[\begin{array}{c}C \\ C A \\ C A^{\mu-1}\end{array}\right]$ are referred to as the controllability matrix of the pair $(A, B)$ and the observability matrix of the pair $(A, C)$, respectively, and have to be of rank $n$ in order that the standard system by controllable (see Theorem 4.9, (4.14)), respectively, observable (see Corollary 4.5, (4.7)).

Remark 4.10. Note from Corollary 4.5 that the rank condition (4.7) requires the necessary condition $\mu \geq[n / s]$ which is then a necessary condition for observability. In the same way, the rank condition (4.15) in Theorem 4.9 requires $\mu \geq[\mathrm{n} / \mathrm{m}]$ which is then a necessary condition for controllability.

\section{Observability and Controllability under Non-Uniform Sampling}

The results of the above section on controllability and observability are now extended for, in general, non-uniform sampling. It has proven in Section 4 that if the standard continuoustime system (i.e., $\alpha=k=1$ ) is controllable/observable then, any fractional system of real order $\alpha$ keeps the corresponding property from that of the standard system. It is now proven that the properties are still kept under non-uniform sampling for almost all choices of the sampling instants provided that their numbers are nonless than the degree $\mu$ of the minimal 
polynomial of the matrix $A$. Such generic choices are possible from the following important property: the linearly independent matrix/vector functions which expand the evolution and control operators defining the state and output trajectory solutions of the Caputo fractional system of order $\alpha$ in polynomial functions of the matrix $A$ in (3.1) are Chebyshev's systems, [4-7].

Theorem 5.1. Assume that the standard system is observable (controllable). Then, the sampled Caputo fractional system of order $\alpha$ is observable (controllable) for almost any choices of $p \geq \mu$ distinct sampling instants subjects to distinct in-between sampling intervals.

Proof. Note from (4.3) and (4.17) that the measurable output of the homogeneous system of the Caputo fractional differential system of order $\alpha$ and the transpose of its forced solution under zero initial conditions at any time instant $t_{i} \in \mathbf{R}_{+}$are, respectively:

$$
\begin{gathered}
y_{\alpha}\left(t_{i}\right)=\bar{\beta}_{\alpha}^{T}\left(t_{i}, p\right) \text { Block Diag }[\operatorname{Ob}(A, C, p) ! \underbrace{k}: \mathbf{O b}(A, C, p)] \bar{x}_{0}, \\
x_{\alpha}^{T}\left(t_{i}\right)=\bar{\gamma}_{u \alpha}^{T}\left(t_{i}, p\right) \operatorname{Co}(A, B, p)^{T},
\end{gathered}
$$

for all $p\left(\in \mathbf{Z}_{+}\right) \geq \mu$, for all $\alpha\left(\in \mathbf{R}_{+}\right) \in[k-1, k]$, with $k \in \mathbf{Z}_{+}$, where $\mathbf{R}^{s \times p k s} \ni \bar{\beta}_{\alpha}^{T}\left(t_{i}, p\right)=$ $\widehat{\beta}_{\alpha}^{T}\left(t_{i}, p\right) I_{s}$, with $\widehat{\beta}_{\alpha}^{T}\left(t_{i}, p\right) \in \mathbf{R}^{1 \times p k}$, and $\bar{\gamma}_{\alpha}^{T}\left(t_{i}, p\right) \in \mathbf{R}^{1 \times p m}$. Now, define

$$
\begin{aligned}
\overline{\mathbf{y}}_{\alpha p}\left(t_{1}, t_{2}, \ldots, t_{p k}\right) & :=\left[y_{\alpha}^{T}\left(t_{1}, p\right), y_{\alpha}^{T}\left(t_{2}, p\right), \ldots, y_{\alpha}^{T}\left(t_{k p}, p\right)\right]^{T} \in \mathbf{R}^{p k s}, \\
\bar{\beta}_{\alpha p}\left(t_{1}, t_{2}, \ldots, t_{p k}\right) & :=\left[\bar{\beta}_{\alpha}^{T}\left(t_{1}, p\right), \bar{\beta}_{\alpha}^{T}\left(t_{2}, p\right), \ldots, \bar{\beta}_{\alpha}^{T}\left(t_{k p}, p\right)\right] \in \mathbf{R}^{p k s \times p k s},
\end{aligned}
$$

for some set of sampling instants $\left\{t_{1}, t_{2}, \ldots, t_{p k}\right\}$; and

$$
\begin{gathered}
\overline{\mathbf{x}}_{\alpha p}\left(t_{1}, t_{2}, \ldots, t_{p k}\right):=\left[x_{\alpha}\left(t_{1}, p\right), x_{\alpha}\left(t_{2}, p\right), \ldots, x_{\alpha}\left(t_{k p}, p\right)\right]^{T} \in \mathbf{R}^{p m \times n}, \\
\bar{\gamma}_{u \alpha p}\left(t_{1}, t_{2}, \ldots, t_{p m}\right):=\left[\boldsymbol{r}_{u \alpha}\left(t_{1}, p\right), \boldsymbol{r}_{u \alpha}\left(t_{2}, p\right), \ldots, \boldsymbol{r}_{u \alpha}\left(t_{p m}, p\right)\right]^{T} \in \mathbf{R}^{p m \times p m},
\end{gathered}
$$

for some set of sampling instants $\left\{t_{1}, t_{2}, \ldots, t_{p m}\right\}$. One gets from (5.3) via (5.1) and (5.4), and from (5.5) via (5.2) and (5.6) the following linear algebraic systems:

$$
\begin{gathered}
\overline{\mathbf{y}}_{\alpha p}\left(t_{1}, t_{2}, \ldots, t_{p k}\right)=\overline{\boldsymbol{\beta}}_{\alpha p}\left(t_{1}, t_{2}, \ldots, t_{p k}\right) \text { Block Diag }[\operatorname{Ob}(A, C, p): \underbrace{k}: \operatorname{Ob}(A, C, p)] \bar{x}_{0}, \\
\overline{\mathbf{x}}_{\alpha p}^{T}\left(t_{1}, t_{2}, \ldots, t_{p k}\right)=\bar{\gamma}_{u \alpha p}\left(t_{1}, t_{2}, \ldots, t_{p m}\right) \operatorname{Co}(A, B, p)^{T} .
\end{gathered}
$$


Note that Block $\operatorname{Diag}[\mathbf{O b}(A, C, p): \underbrace{k}_{\cdots}: \mathbf{O b}(A, C, p)] \in \mathbf{R}^{k p s \times k n}$ is full-rank equal to $k n$ if $p \geq \mu \geq[n / s]$ and $\operatorname{rank} \mathbf{O b}(A, C, p)=\operatorname{rank} \mathbf{O b}(A, C, \mu)=n$ (i.e., if the standard system with $\alpha=k=1$ is observable). A unique solution exists to (5.7) if and only if the set of sampling instants $\left\{t_{1}, t_{2}, \ldots, t_{p k}\right\}$ is chosen so that the square real matrix $\bar{\beta}_{\alpha p}\left(t_{1}, t_{2}, \ldots, t_{p k}\right)$ is nonsingular provided that $\operatorname{rank} \mathbf{O b}(A, C, p)=\operatorname{rank} \mathbf{O b}(A, C, \mu)=n$ since then

$$
\begin{aligned}
& \operatorname{rank}\left[\overline{\mathbf{y}}_{\alpha p}\left(t_{1}, t_{2}, \ldots, t_{p k}\right): \bar{\beta}_{\alpha p}\left(t_{1}, t_{2}, \ldots, t_{p k}\right) \operatorname{Block} \operatorname{Diag}[\operatorname{Ob}(A, C, p): \underbrace{k}_{\cdots}: \mathbf{O b}(A, C, p)]\right] \\
& \quad \times \operatorname{rank}\left[\overline{\boldsymbol{\beta}}_{\alpha p}\left(t_{1}, t_{2}, \ldots, t_{p k}\right) \operatorname{Block} \operatorname{Diag}[\operatorname{Ob}(A, C, p): \underbrace{k}_{\cdots}: \mathbf{O b}(A, C, p)]\right]=k n
\end{aligned}
$$

so that (5.7) is a compatible linear algebraic system (then, the map from $\mathbf{R}^{k n}$ to $\mathbf{R}^{p k s}$ defined via (5.7) is injective) leading to a unique solution $\widehat{\bar{x}}_{0}$ being a least-square estimation of $\bar{x}_{0}$ given by

$$
\widehat{\bar{x}}_{0}=\left(\boldsymbol{\Omega}^{T}\left(t_{1}, t_{2}, \ldots, t_{p k}\right) \boldsymbol{\Omega}\left(t_{1}, t_{2}, \ldots, t_{p k}\right)\right)^{-1} \boldsymbol{\Omega}^{T}\left(t_{1}, t_{2}, \ldots, t_{p k}\right) \overline{\mathbf{y}}_{\alpha p}\left(t_{1}, t_{2}, \ldots, t_{p k}\right),
$$

where

$$
\boldsymbol{\Omega}\left(t_{1}, t_{2}, \ldots, t_{p k}\right):=\overline{\boldsymbol{\beta}}_{\alpha p}\left(t_{1}, t_{2}, \ldots, t_{p k}\right) \operatorname{Block} \operatorname{Diag}[\operatorname{Ob}(A, C, p) \underbrace{k}_{\cdots} \operatorname{Ob}(A, C, p)]
$$

from Rouché-Froebenius theorem for compatibility from Linear Algebra. Then, the observability property is preserved from the standard system for such a set of samples. Also, if $\operatorname{rank} \operatorname{Co}(A, C, p)=\operatorname{rank} \operatorname{Co}(A, C, \mu)=n$ if $p \geq \mu$, so that the standard system is controllable, then

$$
\begin{aligned}
& \operatorname{rank}\left[\overline{\mathbf{x}}_{\alpha p}^{T}\left(t_{1}, t_{2}, \ldots, t_{p k}\right): \bar{\gamma}_{u \alpha p}\left(t_{1}, t_{2}, \ldots, t_{p m}\right) \operatorname{Co}(A, B, p)^{T}\right] \\
& =\operatorname{rank}\left[\bar{r}_{\text {uap }}\left(t_{1}, t_{2}, \ldots, t_{p m}\right) \operatorname{Co}(A, B, p)^{T}\right] \\
& =\operatorname{rank}[\mathbf{C o}(A, B, p)]=\operatorname{rank}[\operatorname{Co}(A, B, \mu)]=n,
\end{aligned}
$$

in (5.8), if and only if the set of sampling instants $\left\{t_{1}, t_{2}, \ldots, t_{p m}\right\}$ is chosen so that the square real matrix $\bar{\gamma}_{\text {uap }}\left(t_{1}, t_{2}, \ldots, t_{p m}\right)$ is nonsingular. Then, the controllability property is preserved from the standard system for such a set of samples. Since the linearly independent real matrix vector functions $\bar{\beta}_{\alpha i}(t, p)$ and linearly independent real vector functions $\gamma_{u \alpha i}^{T}(t, p)$, for 
all $i \in \overline{p-1} \cup\{0\}$, for all $p \geq \mu$ of domain $\mathbf{R}_{0+}$ are also Chebyshev's systems, [4-7] both $\overline{\boldsymbol{\beta}}_{\alpha p}\left(t_{1}, t_{2}, \ldots, t_{p k}\right)$ and $\bar{\gamma}_{\text {uap }}\left(t_{1}, t_{2}, \ldots, t_{p m}\right)$ are nonsingular matrices for almost all choices of the sampling instants.

Remark 5.2. It suffices to choose the sampling periods (i.e. the time intervals in-between any two consecutive sampling instants) in Theorem 5.1 as being mutually distinct and belonging to real intervals of the form $[\eta, \eta+\pi / \omega)$, where $\eta \in \mathbf{R}_{+}$is arbitrary and $\omega$ is an upperbound of the maximum eigenfrequency of (3.1), that is, the maximum absolute value of the imaginary parts of all the complex eigenvalues of $A$, if any. Otherwise, $\omega=+\infty$. This procedure guarantees that the corresponding coefficient matrices of Chebyshev's systems are nonsingular, [4-7]. Some choices are preferred if computation is required. For instance, it can be suitable to solve the linear algebraic system (5.10) so that the coefficient matrix (5.11) be as better conditioned as possible by the choice of the sampling instants. Then, the influence of numerical errors in the computation of the solution is minimized. It can be also suitable to take a number of sampling instants $p$ being large enough to make the estimated initial condition sufficiently close to the real one in the least-squares computation procedure.

For algebraic solvability, it might be possible to reduce the number of sampling instants in (5.7)-(5.8) so that the coefficient matrix of the algebraic systems. The procedure is formalized as follows.

Theorem 5.3. The following properties hold.

(i) Assume that the standard dynamic system is observable. Then, the Caputo fractional linear system of order $\alpha$ is observable under non-uniform sampling as well from a set of existing sampling instants $S T:=\left\{t_{1}, t_{2}, \ldots, t_{\hat{n}}\right\}$ with $\widehat{n}:=\max _{1 \leq i \leq s} n_{i}$ for a set of integers $n_{i} \in \mathbf{Z}_{0+}$ fulfilling $\sum_{i=1}^{S} n_{i}=k n$ in such a way that each ith component of the measured output, that is $y_{\alpha i}\left(t_{i}\right)$, is observed at a set of sampling instants $S T_{i}:=\left\{t_{1}, t_{2}, \ldots, t_{n_{i}}\right\} \subseteq S T$ (which is empty if $\left.n_{i}=0\right)$, for all $i \in \bar{s}$ which fulfils that the coefficient matrix $\widehat{\boldsymbol{\beta}}_{\alpha \hat{n}}\left(t_{1}, t_{2}, \ldots, t_{\hat{n}}\right)$ of $\bar{\beta}$-functions, defined below in the proof, of the associated linear algebraic system of grouped data $\overline{\mathbf{y}}_{\alpha p}\left(t_{1}, t_{2}, \ldots, t_{\hat{n}}\right)$ is nonsingular.

(ii) Assume that the standard dynamic system is controllable. Then, the Caputo fractional linear system of order $\alpha$ is controllable under non-uniform sampling as well from a set of existing sampling instants $S T:=\left\{t_{1}, t_{2}, \ldots, t_{\hat{n}}\right\}$ organized in such a way that for each control component a set of sampling instants $S T_{i}:=\left\{t_{1}, t_{2}, \ldots, t_{n_{i}}\right\} \subseteq S T$ is used, which is empty if (which is empty if $n_{i}=0$ ), for all $i \in \bar{m}$ where $\hat{n}:=\max _{1 \leq i \leq m} n_{i}$ for a set of integers $n_{i} \in \mathbf{Z}_{0+}$ fulfilling $\sum_{i=1}^{m} n_{i}=n$ provided that the associated coefficient matrix is nonsingular.

Proof. (i) For observability under non-uniform sampling with reduced number of samples, the square coefficient matrix of linearly independent matrix functions (5.4), that is $\overline{\boldsymbol{\beta}}_{\alpha p}\left(t_{1}, t_{2}, \ldots, t_{p k}\right)$, of the algebraic linear system of (5.7) may be reduced to a minimum order being the less integer nonless than $k n$ in order to estimate $\bar{x}_{0} \in \mathbf{R}^{k n}$ from a set of samples of $y_{\alpha}(t)$. The associated algebraic problem is formulated so that each component $y_{\alpha i}(t)$ is observed at a set of sampling instants $\left\{t_{i 1}, t_{i 2}, \ldots, t_{i n_{i}}\right\}(i \in \bar{s})$, which can be the empty set for some of the components $j \in \bar{s}$ (and then $n_{j}=0$ ), with a choice of the s nonnegative integers $n_{i}(i \in \bar{s})$ fulfilling the constraint $\sum_{i=1}^{s} n_{i}=k n$. Since the functions in the coefficient matrix are a Chebyshev's system, the resulting coefficient matrix may be generically constructed as being nonsingular for a set of sampling instants $\left\{t_{1}, t_{2}, \ldots, t_{\hat{n}}\right\}$ selected as $t_{i j}\left(j \in \bar{n}_{i}\right)=t_{j}$ if 
$j \in \bar{n}_{i}$ and $\bar{n}_{i} \leq j$, for all $i \in \bar{s}$, for all $j \in \widehat{n}$ with $\widehat{n}:=\max _{1 \leq i \leq s} n_{i}$. Equation (5.1) is replaced by the component-to-component corresponding set of equations

$$
y_{\alpha i}\left(t_{i}\right)=\bar{\beta}_{\alpha i}^{T}\left(t_{j}, j \in \bar{n}_{i}\right) G_{i} \bar{x}_{0} ; \quad \forall i \in \bar{s}
$$

where

$$
G_{i}:=\operatorname{Block} \operatorname{Diag}[\operatorname{Ob}\left(A, C_{i}^{T}, \mu\right) \underbrace{\cdots}: \operatorname{Ob}\left(A, C_{i}^{T}, \mu\right)] ; \quad \forall i \in \bar{s}
$$

so that the whole algebraic system (5.7) is replaced with

$$
\overline{\mathbf{y}}_{\alpha p}\left(t_{1}, t_{2}, \ldots, t_{\hat{n}}\right)=\widehat{\boldsymbol{\beta}}_{\alpha \hat{n}}\left(t_{1}, t_{2}, \ldots, t_{\hat{n}}\right) \text { Block Diag }[G_{1}: \underbrace{s}: G_{S}] \bar{x}_{0}
$$

where

$$
\widehat{\boldsymbol{\beta}}_{\alpha \widehat{n}}\left(t_{1}, t_{2}, \ldots, t_{\hat{n}}\right):=\operatorname{Block} \operatorname{Diag}\left[\left[\bar{\beta}_{\alpha 1}^{T}\left(t_{j}, j \in \bar{n}_{1}\right) \vdots \ldots:\right] \bar{\beta}_{\alpha s}^{T}\left(t_{j}, j \in \bar{n}_{s}\right)\right] .
$$

Then, the mapping from $\mathbf{R}^{k n} \rightarrow \mathbf{R}^{k n}$ defined from (5.15) is injective if and only if $\operatorname{rank} \operatorname{Ob}(A, C, \mu)=n$, requiring $s \geq[\mu / n]$, and, furthermore, the set of sampling instants $\left\{t_{1}, t_{2}, \ldots, t_{\hat{n}}\right\}$ is chosen in such a way that $\operatorname{Det} \widehat{\boldsymbol{\beta}}_{\alpha \widehat{n}}\left(t_{1}, t_{2}, \ldots, t_{\widehat{n}}\right) \neq 0$. Infinitely, many such sets of sampling instants always exist from the property of Chebyshev's systems of the functions defining $\widehat{\boldsymbol{\beta}}_{\alpha \widehat{n}}\left(t_{1}, t_{2}, \ldots, t_{\widehat{n}}\right)$.

(ii) The proof is similar to that of property (i), and then its details are omitted. The guidelines for controllability under non-uniform sampling with a reduced number of sampling instants are as follows. One proceeds in a closed way the square coefficient matrix (5.6), that is $\bar{\gamma}_{\text {uap }}\left(t_{1}, t_{2}, \ldots, t_{p m}\right)$, of the algebraic linear system of (5.8) with a set of nonnegative integers $n_{i}(i \in \bar{m})$, related to the input components, satisfying $\sum_{i=1}^{m} n_{i}=n$ and a set of sampling instants $\left\{t_{1}, t_{2}, \ldots, t_{\hat{n}}\right\}$ selected as $t_{i j}\left(j \in \bar{n}_{i}\right)=t_{j}$ if $j \in \bar{n}_{i}$ and $\bar{n}_{i} \leq j$, for all $i \in \bar{m}$, for all $j \in \widehat{n}$ with $\widehat{n}:=\max _{1 \leq i \leq m} n_{i}$.

Remark 5.4. It follows from Corollaries 4.4, and 4.5 and Remark 4.10 that the controllability and observability of the continuous-time system are independent of the positive real fractional order $\alpha$, that is, those properties hold for the fractional case if they hold for the standard one. Those conditions are necessary conditions for controllability/observability under non-uniform sampling (see Theorems 5.1 and 5.3 and Remark 5.2) which hold for generic choices of always existing sampling instants such that the appropriate coefficient matrices are nonsingular. Thus, we can conclude that if the standard system is controllable (resp., observable), then they always exist generically chosen distinct sampling periods in any number exceeding the degree of the minimal polynomial of the matrix $A$ such that the controllability (resp., observability) are maintained under such a non-uniform sampling sets. 
The method used to investigate the controllability and observability properties under nonuniform sampling is close to that used in [8], namely, first to characterize the fulfilment of the corresponding property for the continuous-time case as a necessary condition, as expected, then to establish a sufficiency-type condition on a certain coefficient matrix, depending on the sampling instants, so as to ensure that the corresponding property holds under non-uniform sampling. Both conditions are of full-rank matrix type and then easily testable. The condition on the sampling instants admits a rather generic choices of them from a well-known property of Chebyshev's systems, [5,6]. A potential way of choosing the set of sampling periods is via optimizing or suboptimizing the condition number of the coefficient matrix of the associate linear algebraic system. This can be accompanied of taking a sufficiently number of data from a sufficiently large set of sampling instants exceeding the degree of the minimal polynomial of the relevant coefficient matrix. In that way, the effects of the transmission of potential errors from data and parameters to results in the controllability and observability problems can become improved, $[6-8,18]$.

\section{An Example}

Consider an ideal DC motor of normalized quotient of payload inertia and gain with a second-order transfer function $G(s)=1 / s(s+1)$ at a fractional order $\alpha=1$, that is an standard system, where $s$ is the Laplace transform argument. This transfer function also describes the step-response of a closed-loop transfer function $1 / s$ under unit feedback and zero initial conditions, [2]. Assume that such a transfer function is changed to $G_{0}(s)=s^{-4 / 3}$ so that step response is independent of the payload inertia at a fractional order setting of $\alpha=4 / 3$, equivalently, we have a second order transfer function $G(s)=1 / s\left(s^{4 / 3}+1\right)$. A brief discussion of such a transfer function under a controllability point of view follows. For fractional order $\alpha=1$ (i.e., for the standard system), the dynamic system associated with it, which is an equivalent model in state-space, is defined by the 2nd-order continuous-time differential system for any piecewise continuous control function $u: \mathbf{R}_{0+} \rightarrow \mathbf{R}_{0+}$ as follows:

$$
\left[\begin{array}{c}
\dot{x}_{1}(t) \\
\dot{x}_{2}(t)
\end{array}\right]=\left[\begin{array}{cc}
0 & 1 \\
0 & -1
\end{array}\right]\left[\begin{array}{l}
x_{1}(t) \\
x_{2}(t)
\end{array}\right]+\left[\begin{array}{l}
0 \\
1
\end{array}\right] u(t) ; \quad\left[\begin{array}{l}
x_{1}(0) \\
x_{2}(0)
\end{array}\right] \in \mathbf{R}^{2},
$$

where the output of the transfer function is $y(t)=x_{1}(t)$. The controllability matrix of the above system is $\left[\begin{array}{cc}0 & 1 \\ 1 & -1\end{array}\right]$ having rank two so that the standard continuous-time system $(\alpha=1)$ is controllable. Then, the necessary condition of controllability for any positive real fractional order $\alpha>0$ holds. The solution for $x_{1}(t)$ for zero initial conditions at $t_{0}=0$ and unit step (Heaviside's function) control is given as follows from (3.1)-(3.4) through the two-real positive parameters Mittag-Leffler function defined by $E_{\alpha \beta}(z)=\sum_{\ell=0}^{\infty}\left(z^{\ell} / \Gamma(\alpha \ell+\beta)\right)$, [2]:

$$
\begin{aligned}
x_{1}(t) & =L^{-1}\left\{\frac{1}{s}-\frac{s^{4 / 3-1}}{s^{4 / 3+1}}\right\}=1-E_{4 / 3}\left(-t^{-4 / 3}\right)=t^{4 / 3} E_{4 / 3,4 / 3+1}\left(-t^{4 / 3}\right) \\
& =\frac{t^{4 / 3}}{\Gamma(4 / 3+1)}-\frac{t^{8 / 3}}{\Gamma(8 / 3+1)}+\frac{t^{4}}{\Gamma(5)}-\cdots, \\
x_{2}(t) & =\frac{4}{3} \frac{t^{1 / 3}}{\Gamma(4 / 3+1)}-\frac{8}{3} \frac{t^{5 / 3}}{\Gamma(8 / 3+1)}+\frac{4 t^{3}}{\Gamma(5)}-\cdots,
\end{aligned}
$$


where $L$ denotes the Laplace transform operator of inverse $L^{-1}$. Since the functions $\beta_{(\cdot)}$ in (3.3)-(3.4) used in the expansions of the evolution operator and in that of its forced counterpart in the forced response of the system are linearly independent and also Chebyshev's systems (see Lemma 3.2, Theorem 5.1, and Remark 5.4), it follows that the controllability of the system of fractional order $4 / 3$ is guaranteed under (in general) nonuniform sampling for any sets consisting of any two or more distinct sampling instants subject to distinct sampling intervals. The property holds as well for any other real fractional order $\alpha>0$. For instance, take null initial conditions at $t_{0}=0$ and two sampling instants $t_{2}\left(=t_{1}+T_{1}\right)>t_{1}\left(=T_{0}\right)>0$ of associate sampling periods $T_{1}=t_{2}-t_{1}, T_{0}=t_{1}$ and some piecewise constant control $u(t)=u_{0}$; for all $t \in\left[0, t_{1}\right)$ and $u(t)=u_{1}$, for all $t \in\left[t_{1}, t_{2}\right)$. Then,

$$
\begin{aligned}
x_{1}\left(t_{2}\right)= & \left(\frac{T_{0}^{4 / 3}}{\Gamma(4 / 3+1)}-\frac{T_{0}^{8 / 3}}{\Gamma(8 / 3+1)}+\frac{T_{0}^{4}}{\Gamma(5)}-\cdots\right) u_{1} \\
& +\left(\frac{T_{1}^{4 / 3}}{\Gamma(4 / 3+1)}-\frac{T_{1}^{8 / 3}}{\Gamma(8 / 3+1)}+\frac{T_{1}^{4}}{\Gamma(5)}-\cdots\right) u_{0}, \\
x_{2}\left(t_{2}\right)= & \left(\frac{4}{3} \frac{T_{0}^{1 / 3}}{\Gamma(4 / 3+1)}-\frac{8}{3} \frac{T_{0}^{5 / 3}}{\Gamma(8 / 3+1)}+\frac{4 T_{0}^{3}}{\Gamma(5)}-\cdots\right) u_{1} \\
& +\left(\frac{4}{3} \frac{T_{1}^{1 / 3}}{\Gamma(4 / 3+1)}-\frac{8}{3} \frac{T_{1}^{5 / 3}}{\Gamma(8 / 3+1)}+\frac{4 T_{1}^{3}}{\Gamma(5)}-\cdots\right) u_{0} .
\end{aligned}
$$

Thus, note that the coefficient matrix of the above linear algebraic system from the unknown vector $\left(u_{0}, u_{1}\right)^{T}$ to de data $\left(x_{1}\left(t_{2}\right), x_{2}\left(t_{2}\right)\right)^{T}$ at $t=T_{2}$ is nonsingular if $T_{0} \neq T_{1}$ guaranteeing the sufficiency-type condition of guaranteeing the controllability of the continuous-time system under non-uniform sampling. By choosing sets of more sampling instants and associated sampling periods exceeding two, the same conclusion is achievable by constructing fullcolumn rank coefficient matrices. A close reasoning would apply for any other positive fractional order.

\section{Acknowledgments}

The author is grateful to the Spanish Ministry of Education for its partial support of this work through Grant no. DPI2009-07197. He is also grateful to the Basque Government by its support through Grants no. GIC07143-IT-269-07 and SAIOTEK S-PE09UN12. He is also grateful to the reviewers by their useful suggestions and comments.

\section{References}

[1] A. A. Kilbas, H. M. Srivastava, and J. J. Trujillo, Theory and Applications of Fractional Differential Equations, vol. 204 of North-Holland Mathematics Studies, Elsevier Science, Amsterdam, The Netherlands, 2006.

[2] S. Das, Functional Fractional Calculus for System Identification and Controls, Springer, Berlin, Germany, 2008. 
[3] M. De la Sen, "Some results on the solutions of Caputo fractional linear time-invariant systems of any order with internal point delays," http://arxiv.org/abs/1009.4311.

[4] A. F. Timan, Theory of Approximation of Functions of a Real Variable, Pergamon Press, New York, NY, USA, 1963.

[5] J. L. Malaina and M. de la Sen, "Extended Chebyshev systems for the expansions of exp(At)," Collectanea Mathematica, vol. 40, no. 3, pp. 197-216, 1989.

[6] I. Troch, "Sampling with arbitrary choice of the sampling instants," Automatica, vol. 9, no. 1, pp. 117$124,1973$.

[7] M. de la Sen, "Application of the nonperiodic sampling to the identifiability and model matching problems in dynamic systems," International Journal of Systems Science, vol. 14, no. 4, pp. 367-383, 1983.

[8] M. De la Sen, "On the properties of reachability, observability, controllability, and constructibility of discrete-time positive time-invariant linear systems with aperiodic choice of the sampling instants," Discrete Dynamics in Nature and Society, vol. 2007, Article ID 84913, 23 pages, 2007.

[9] A. Viswanathan, A. Gelb, D. Cochran, and R. Renaut, “On reconstruction from non-uniform spectral data," Journal of Scientific Computing, vol. 45, no. 1-3, pp. 487-513, 2010.

[10] H. Xu, "Uniform exponential convergence of sample average random functions under general sampling with applications in stochastic programming," Journal of Mathematical Analysis and Applications, vol. 368, no. 2, pp. 692-710, 2010.

[11] G. H. Zou, Y. F. Li, R. Zhu, and Z. Guan, "Imputation of means of ratios for missing data and its application to PPSWR sampling," Acta Mathematica Sinica, vol. 26, no. 5, pp. 863-874, 2010.

[12] K. I. Koike, "Sequential estimation procedures for end points of support in a non-regular distribution," Communications in Statistics-Theory and Methods, vol. 39, no. 8-9, pp. 1585-1596, 2010.

[13] B. Beahan and W. Cowley, "Improving frequency estimation performance for burst transmissions by optimising reference symbol distribution," Signal Processing, vol. 90, no. 12, pp. 3037-3045, 2010.

[14] G. Zhao, G. Shi, and J. Zhou, "Angel estimation via frequency diversity of the SIAR radar based on Bayesian theory," Science China Technological Sciences, vol. 53, no. 9, pp. 2581-2588, 2010.

[15] M. Miskowicz, "Efficiency of event-based sampling according to error energy Criterion," Sensors, vol. 10, no. 3, pp. 2242-2261, 2010.

[16] L. Fesquet and B. Bidégaray-Fesquet, "IIR digital filtering of non-uniformly sampled signals via state representation," Signal Processing, vol. 90, no. 10, pp. 2811-2821, 2010.

[17] K. P. M. Bhat and H. N. Koivo, "Model characterizations of controllability and observability in time delay systems," IEEE Transactions on Automatic Control, vol. 21, no. 2, pp. 292-293, 1976.

[18] M. de la Sen, "Adaptive sampling for improving the adaptation transients in hybrid adaptive control," International Journal of Control, vol. 41, no. 5, pp. 1189-1205, 1985. 


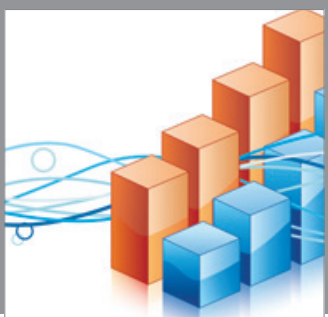

Advances in

Operations Research

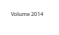

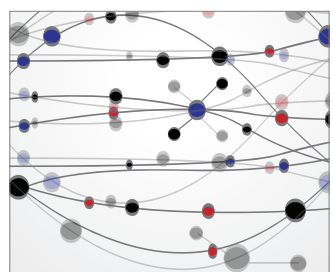

\section{The Scientific} World Journal
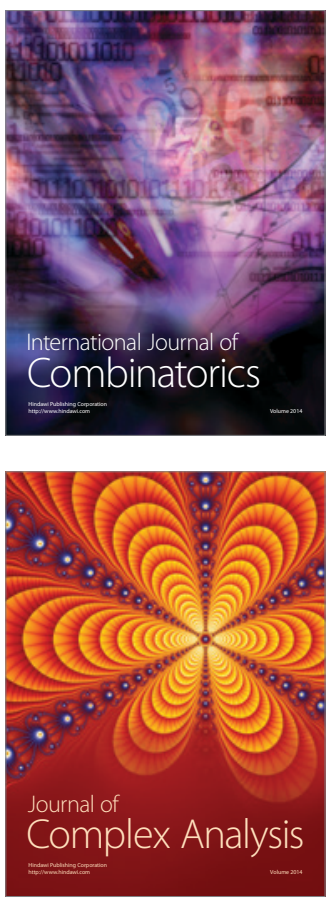

International Journal of

Mathematics and

Mathematical

Sciences
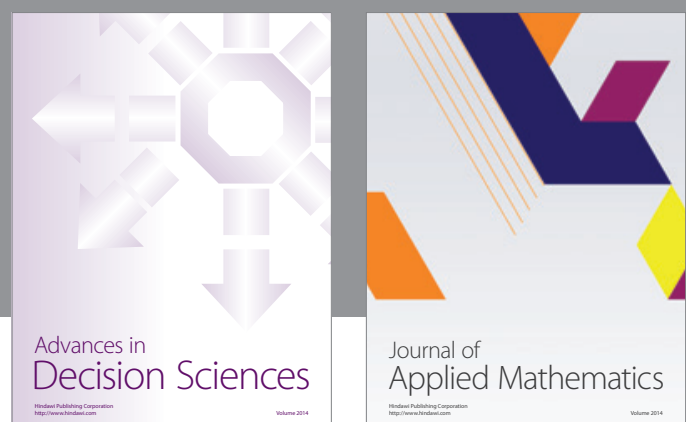

Journal of

Applied Mathematics
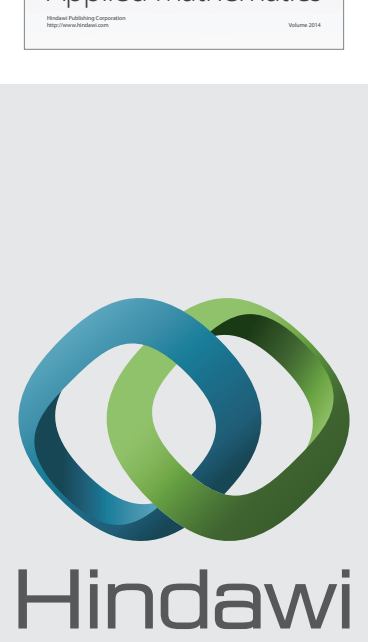

Submit your manuscripts at http://www.hindawi.com
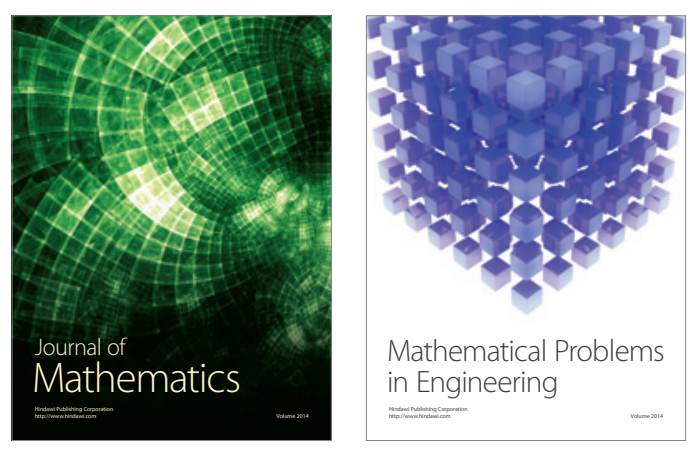

Mathematical Problems in Engineering
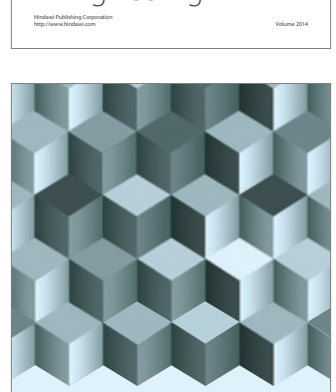

Journal of

Function Spaces
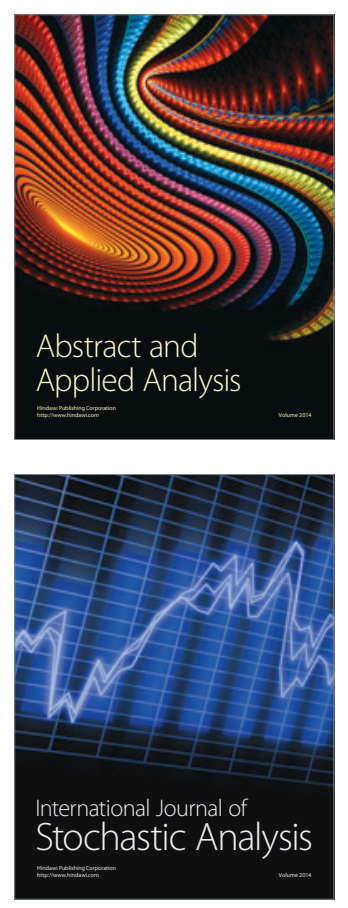

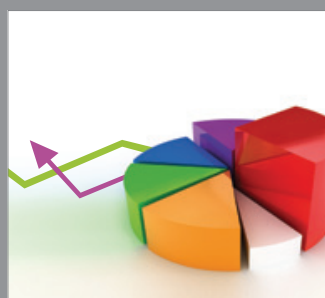

ournal of

Probability and Statistics

Promensencen
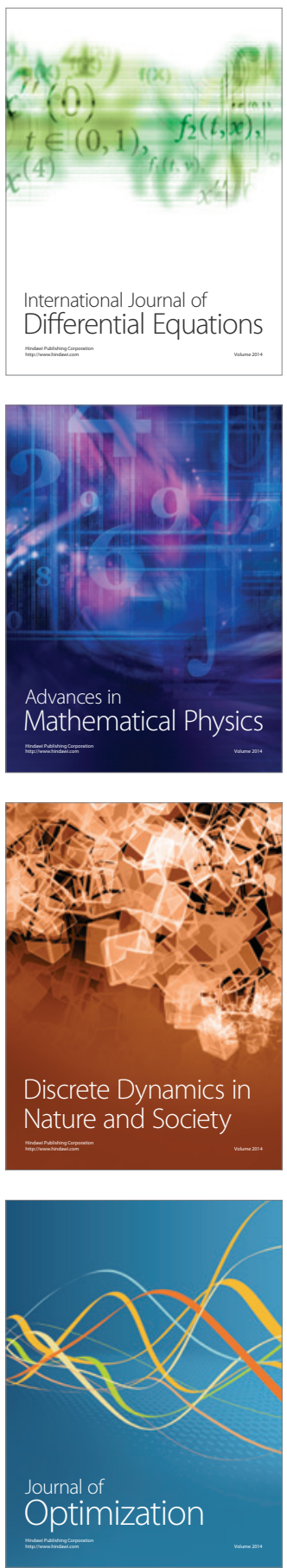